\title{
Decentralized Formation Pose Estimation for Spacecraft Swarms
}

\author{
Kai Matsuka ${ }^{\mathrm{a}, *}$, Aaron O. Feldman ${ }^{\mathrm{a}}$, Elena S. Lupu ${ }^{\mathrm{a}}$, Soon-Jo Chung ${ }^{\mathrm{a}, \mathrm{b}}$, Fred \\ Y. Hadaegh ${ }^{b}$ \\ ${ }^{a} 1200$ E. California Blvd, Pasadena, CA, USA 91125 \\ ${ }^{b} 4800$ Oak Grove Dr, Pasadena, CA, USA 91109
}

\begin{abstract}
For spacecraft swarms, the multi-agent localization algorithm must scale well with the number of spacecraft and adapt to time-varying communication and relative sensing networks. In this paper, we present a decentralized, scalable algorithm for swarm localization, called the Decentralized Pose Estimation (DPE) algorithm. The DPE considers both communication and relative sensing graphs and defines an observable local formation. Each spacecraft jointly localizes its local subset of spacecraft using direct and communicated measurements. Since the algorithm is local, the algorithm complexity does not grow with the number of spacecraft in the swarm. As part of the DPE, we present the Swarm Reference Frame Estimation (SRFE) algorithm, a distributed consensus algorithm to coestimate a common Local-Vertical, Local-Horizontal (LVLH) frame. The DPE combined with the SRFE provides a scalable, fully-decentralized navigation solution that can be used for swarm control and motion planning. Numerical simulations and experiments using Caltech's robotic spacecraft simulators are presented to validate the effectiveness and scalability of the DPE algorithm.
\end{abstract}

Keywords: swarm localization, spacecraft swarm, large scale estimation, and decentralized estimation.

\section{Introduction}

Spacecraft swarms have the potential to revolutionize the space industry by enabling missions such as distributed aperture telescopes, space structure assemblies, and cooperative deep space explorations (Chung and Hadaegh, 2011. Cash, 2006, Gdoutos et al., 2018). These multi-spacecraft missions have several advantages over monolithic satellite missions, such as robustness to individual spacecraft loss and improved science return (Hadaegh et al., 2016; Brown et al., 2009). Since ground-in-the-loop control of individual spacecraft is prohibitive

${ }^{*}$ Corresponding author

Preprint submitted to Advances in Space Research

June 10, 2020 
for swarm missions, the spacecraft swarm must autonomously estimate their states and maintain their formation.

An essential prerequisite for swarm motion planning and collision-avoidance algorithms (Morgan et al., 2016) is swarm localization. Swarm localization is a multi-agent localization problem where the number of agents is so large that no single agent can maintain the information for all of the agents in the swarm. Swarm localization is challenging for several reasons. First, in some multi-agent localization algorithms for small-scale swarms, the time complexity scales at least linearly with the formation size (Kamal et al. 2012, Açıkmeşe et al. 2014). Hence, these algorithms are not suitable for large-scale swarms.

Another challenge is the requirement that each spacecraft must estimate the absolute orbit of a reference spacecraft in order to define a common LocalHorizontal, Local-Vertical (LVLH) frame estimate (see Figure 1). This estimation is challenging for large-scale swarms as some of the spacecraft in the swarm may not make a direct measurement of this reference spacecraft (Chung et al. 2018, Bandyopadhyay and Chung, 2018). Any algorithm suitable for swarm localization requires a novel approach that explicitly addresses these challenges.

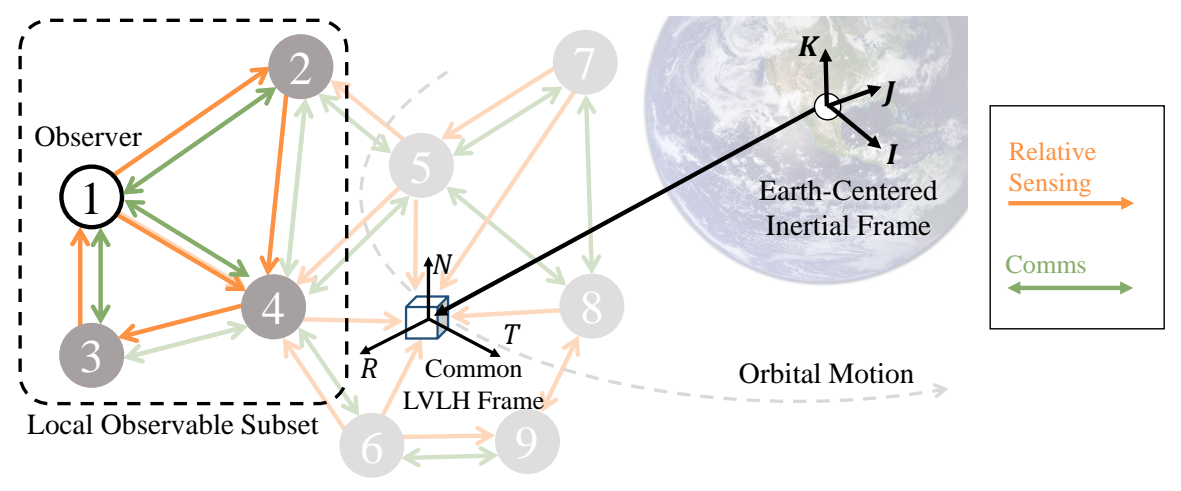

Figure 1: A spacecraft swarm and its relative sensing and communication networks. The DPE estimates the spacecraft poses in the local observable subset with respect to the common Local-Horizontal, Local-Vertical (LVLH) frame.

In this paper, we solve the swarm localization problem by proposing a local algorithm called the Decentralized Pose Estimation (DPE) algorithm. Using the DPE, each spacecraft estimates the states of only a local subset of the swarm. Neighbor spacecraft cooperate to improve the pose estimates by sharing their measurements. The DPE uses the result of nonlinear observability analysis to determine the local observable subset of spacecraft given the ad hoc relative sensing and communication graphs for each spacecraft. Then it jointly localizes the spacecraft in the local observable subset by fusing the measurements that are collected over the communication network. The DPE offers advantage over the standard pose estimation without communication by improving the estimation accuracy and by increasing the number of observable spacecraft. For our 
specific implementation, we represent the attitude of spacecraft with quaternion while the Extended Kalman Filter (EKF) estimates the error attitude state in minimal coordinate at each time step. As part of the DPE, we also present the Swarm Reference Frame Estimation (SRFE) algorithm, which allows each spacecraft to co-estimate the common LVLH frame of the swarm in a decentralized manner. The SRFE applies the decentralized consensus filter (Kamal et al. 2013 Bandyopadhyay and Chung, 2018) to estimate the reference spacecraft that may be visible to only a subset of the spacecraft. The DPE combined with the SRFE provides a fully decentralized navigation solution that can be used in swarm motion planning.

The DPE algorithm was verified in simulations and real-time robotic experiments. The DPE performance was compared against that of an Individual EKF, wherein each spacecraft uses only its measurements to estimate only those spacecraft it directly measures, and a Centralized EKF, which has access to all the information in the swarm. The robotic experiment was conducted on Caltech's robotic spacecraft dynamics simulators, the Multi-Spacecraft Testbed for Autonomy Research (M-STAR) (Nakka et al., 2018; Foust et al., 2018). The relative poses of spacecraft were estimated using vision on-board each spacecraft. We validated the DPE estimate against the ground truth obtained from a motion capture system. In summary, this paper presents a scalable, decentralized algorithm for swarm localization that is appropriate for on-board implementation.

\subsection{Related Work}

The literature review is divided into the following parts: the multi-agent localization problem for a team of robotic systems, some domain-specific largescale estimation in other applications, estimation of the common reference frame for a swarm, and experimental validation of vision-based relative pose estimation algorithms.

The research in multi-agent localization, a problem of estimating poses of multiple robots in a group, has advanced over the years in the robotics community as interest in multi-agent coordination (Yan et al. 2013) has increased. While there already exists a variety of multi-agent localization algorithms in the literature, they predicate on assumptions specific to the respective applica-

tion. Some research assumes that the team of agents rendezvous infrequently and that the robots exchange information upon close encounters, such as Multiagent Simultaneous Localization and Mapping (Fox et al., 2000; Carlone et al. 2010, Zhou and Roumeliotis, 2006, Aragues et al., 2011). In contrast, other research on multi-agent localization assumes that the team of robots maintains communication links over a possibly time-varying network (Martinelli et al., 2005, Franchi et al. 2013, Roumeliotis and Bekey, 2002). Some prior work explicitly considers joint pose estimation over a network of relative measurements (Martinelli et al., 2005: Marelli and Fu, 2015, Kekatos and Giannakis, 2012, Blackmore and Hadaegh, 2009 Bezouska and Barnhart, 2019). However, this work assumes all-to-all communication, so it is not scalable for swarm applications. In another perspective, multi-agent localization can be seen as a 
distributed estimation problem where the states to be estimated are the poses of the agents themselves. For a small-scale estimation problem, decentralized consensus filters have been shown to be highly effective (Kamal et al., 2013. Bandyopadhyay and Chung, 2018). However, these algorithms require all of the nodes to have the same set of states. Since swarm localization is a large-scale estimation problem, these algorithms also do not scale well with the number of robots in the formation.

Large-scale estimation can be made tractable by limiting each node to estimate only some subset of the swarm. In the context of networked power systems, this problem has been studied for recursive estimation of static states (Kekatos and Giannakis, 2012, Marelli and Fu, 2015). Another proposed decentralized local algorithm addressed dynamical systems with an L-banded network structure (Khan and Moura, 2008), such as dynamics governed by partial differential equations. While this approach yields a near-optimal result for the specific problem, the problem assumes that each agent has a predetermined ordering of the graph nodes. This is not valid for swarm localization problems where the network topology is ad hoc. A scalable swarm localization algorithm requires a new approach in which local estimation accommodates ad hoc networks in terms of communication and relative sensing.

A separate but critical sub-component for swarm localization is the estimation of a common reference frame for the swarm. Motion planning algorithms typically require a local reference frame with respect to which the swarm poses are defined. Some multi-agent robotics literature discusses the estimation of a common reference frame (Nagavalli et al., 2014, Franceschelli and Gasparri, 2013: López-Limón et al., 2014). For formation flying spacecraft applications, the goal of the common reference frame estimation is to obtain knowledge of the absolute orbital state. The estimated state vector is small, so the decentralized consensus filter (Kamal et al., 2013, Bandyopadhyay and Chung, 2018) can be applied to solve this problem.

Some previous literature on vision-based relative pose estimation involves the implementation of the proposed algorithm on hardware. While there are few in-flight experiments for vision-based relative navigation (Fourie et al. 2014 ), most of the vision-based pose estimation algorithms were validated through simulations (Capuano et al., 2020) or on the ground using high-fidelity testbeds. For example, some research work studies the pose estimation for a cooperative single target (Romano et al., 2007, Tweddle and Saenz-Otero, 2015; Zhang et al. 2014) or an uncooperative one (De Jongh et al., 2020). However, there has not been an experimental validation on a vision-based pose estimation for spacecraft swarms.

\subsection{Contributions}

In this paper, we present a novel, decentralized swarm localization algorithm called the Decentralized Pose Estimation (DPE) algorithm. The main contributions of this paper are as follows. First, the DPE estimates the states of only a local subset of spacecraft, so the algorithm complexity on each spacecraft

does not grow with the swarm size. Based on the ad hoc relative sensing and 
communication graphs, the DPE uses the observability criteria to determine the set of observable spacecraft. Second, we provide a strategy for estimating the common reference frame using a distributed consensus filter wherein we apply the decentralized consensus filter for common LVLH frame estimation for spacecraft swarms. We validated the algorithm in numerical simulation and experiments. To the authors' knowledge, the experimental validation of a cooperative pose estimation algorithm using a swarm of spacecraft simulators equipped with electro-optical sensors is also novel.

This paper extends our previous work (Matsuka et al. 2019) in the following ways:

- we modified the DPE such that the estimated states are poses with respect to a common LVLH frame and the new measurement model includes absolute pose measurements available from the spacecraft's communication neighbors,

- we formulated the DPE for full six degrees-of-freedom, rather than the planar dynamics considered in (Matsuka et al., 2019),

- we developed the SRFE algorithm to estimate a common LVLH frame using the decentralized consensus filter,

- we provided more thorough numerical analyses illustrating the advantages of the DPE,

- we experimentally validated the algorithm in time-varying relative sensing and communication networks.

The outline of the remainder of the paper is as follows. We first define notations and review the relative orbital dynamics in Section 2. Next, we describe the DPE algorithm in detail in Section 3 and the SRFE algorithm in Section 4 Then we describe the numerical simulations in Section 5 and the robotic experiment results in Section 6. The conclusion is presented in Section 7 .

\section{Preliminaries}

Let $\mathcal{G}^{\mathrm{s}}=\left(\mathcal{V}, \mathcal{E}^{\mathrm{s}}\right)$ denote a directed graph that describes the relative sensing graph, with $\mathcal{V}=\{1, \ldots, N\}$ the set of spacecraft and $\mathcal{E}^{\text {s }}$ the set of edges. An edge $(i, j)$ is in $\mathcal{E}^{\mathrm{s}}$ when the $i$-th spacecraft measures the relative pose of the $j$-th spacecraft. Similarly, let $\mathcal{G}^{\mathrm{c}}=\left(\mathcal{V}, \mathcal{E}^{\mathrm{c}}\right)$ denote the communication graph, an undirected graph for the communication topology. We say $(i, j) \in \mathcal{E}^{\mathrm{c}}$ if there is a communication link between the $i$-th and the $j$-th spacecraft. Note that the measurement graph and the communication graph may be different in general. The out-neighbors of a node $i$ in a graph $\mathcal{G}$ are defined as $\mathcal{N}_{i}=\{j \in \mathcal{V} \mid(i, j) \in$ $\mathcal{E}(\mathcal{G})\}$ and we use subscripts $s$ and $c$ to distinguish the neighbors for relative sensing and communication graphs respectively. The neighborhood of node $i$ is defined as $\overline{\mathcal{N}}_{i}=\mathcal{N}_{i} \cup\{i\}$. The degree of a node in a graph is defined as $d_{i}=\sum_{j=1}^{N} A_{i j}$ where $A$ is the adjacency matrix of the graph and the maximum degree $\Delta$ of a graph is defined as $\Delta=\max \left(d_{i}\right)$.

A column concatenation of vectors $\mathbf{x}_{1}, \ldots, \mathbf{x}_{n}$ is written as $\overline{\mathbf{x}}=\left[\mathbf{x}_{1} ; \ldots ; \mathbf{x}_{n}\right]$ or $\overline{\mathbf{x}}=\|_{i=1, \ldots, n} \mathbf{x}_{i}$, where the bar over the variable denotes an augmented variable defined as a concatenation of variables. The position and velocity of an 
object $a$ in frame $b$ are denoted $\mathbf{p}_{a, b}, \mathbf{v}_{a, b}$. The attitude and angular rate of the frame $a$ with respect to the frame $b$ are denoted $\mathbf{q}_{a, b}$ and $\boldsymbol{\omega}_{a, b}$. The function $R(\cdot)$ maps a quaternion onto a rotation matrix such that $\mathbf{x}_{b}=R\left(\mathbf{q}_{a, b}\right) \mathbf{x}_{a}$ where $\mathbf{x}_{a}, \mathbf{x}_{b}$ are vectors expressed in frame $a$ and $b$ respectively. We use ${ }^{\wedge}$ above variables to denote the expectation.

The relative states of formation flying spacecraft are defined using a localvertical, local-horizontal (LVLH) frame that is attached to the common reference spacecraft as shown in Figure 1 The LVLH frame, denoted by subscript $L$, is defined as follows: the $x$ direction, $R$, is along the orbit position vector to the spacecraft; the $z$ direction, $N$, is along the angular momentum vector of the spacecraft's orbit; and the $y$ direction, $T$, completes the right-handed coordinate system. The origin of the LVLH frame coincides with the center of gravity of the reference spacecraft.

\subsection{Relative Orbital Dynamics}

This section reviews the equation of motion for the relative orbital dynamics. For the rest of the paper, we assume that each spacecraft is in a near-circular orbit, there are no perturbations and that all the spacecraft are in proximity such that the relative orbital dynamics can be linearized to the Hill-ClohessyWiltshire (HCW) equations (Schaub and Junkins, 2005). There exist some dynamics models that include eccentricity or other perturbation effects (Yamanaka and Ankersen, 2002: Morgan et al., 2012, Sullivan et al., 2017); however, we choose the HCW model dynamics to illustrate more clearly the decentralized aspects of the algorithm. Suppose $\mathbf{p}_{i, L}, \mathbf{v}_{i, L}$ are the position and velocity vectors of the $i$-th spacecraft with respect to an LVLH frame that is commonly known among all the spacecraft in the swarm. The dynamics of translational states using the $\mathrm{HCW}$ equations are described by

$$
\left[\begin{array}{c}
\dot{\mathbf{p}}_{i, L} \\
\dot{\mathbf{v}}_{i, L}
\end{array}\right]=\mathbf{A}_{t}\left[\begin{array}{c}
\mathbf{p}_{i, L} \\
\mathbf{v}_{i, L}
\end{array}\right]+\mathbf{B}_{t} \mathbf{w}_{t}
$$

where state matrix $\mathbf{A}_{t}$ and actuation matrix $\mathbf{B}_{t}$ are given by

$$
\begin{aligned}
\mathbf{A}_{t}=\left[\begin{array}{cc}
\mathbf{0}_{3 \times 3} & \mathbf{I}_{3} \\
\mathbf{A}_{v p} & \mathbf{A}_{v v}
\end{array}\right], & \mathbf{B}_{t}=\left[\begin{array}{c}
\mathbf{0}_{3 \times 3} \\
\mathbf{I}_{3}
\end{array}\right] \\
\mathbf{A}_{v p}=\left[\begin{array}{ccc}
3 n^{2} & 0 & 0 \\
0 & 0 & 0 \\
0 & 0 & -n^{2}
\end{array}\right], & \mathbf{A}_{v v}=\left[\begin{array}{ccc}
0 & 2 n & 0 \\
-2 n & 0 & 0 \\
0 & 0 & 0
\end{array}\right]
\end{aligned}
$$

where $n$ is the mean anomaly of the reference spacecraft orbit and $\mathbf{w}_{t} \sim$ $\mathcal{N}\left(\mathbf{0}_{3 \times 1}, \mathbf{W}_{t}\right)$ is assumed to be a zero mean Gaussian process noise.

\subsection{Attitude Dynamics}

For specific implementation of the DPE, we choose quaternions to represent the attitude components of the spacecraft state (Markley and Crassidis, 2014). A quaternion is defined as $\mathbf{q}=\left[\mathbf{q}_{v} ; q_{s}\right]$ where $\mathbf{q}_{v} \in \mathbb{R}^{3}$ and $q_{s} \in \mathbb{R}$ are the vector 
and scalar components of the quaternion, respectively. A unit quaternion $\mathbf{q} \in S^{3}$ satisfies the constraint $\mathbf{q}^{\top} \mathbf{q}=1$. Quaternion multiplication is denoted with the group operator $\otimes$ and is defined as

$$
\mathbf{q}^{\prime} \otimes \mathbf{q}=\left[\begin{array}{c}
q_{s}^{\prime} \mathbf{q}_{v}+q_{s} \mathbf{q}_{v}^{\prime}-\mathbf{q}_{v}^{\prime} \times \mathbf{q}_{v} \\
q_{s}^{\prime} q_{s}-\mathbf{q}_{v}^{\prime} \cdot \mathbf{q}_{v}
\end{array}\right]
$$

The inverse of a quaternion is defined as

$$
\mathbf{q}^{-1}=\frac{1}{\|\mathbf{q}\|}\left[\begin{array}{c}
-\mathbf{q}_{v} \\
q_{s}
\end{array}\right] .
$$

A small attitude perturbation $\delta \mathbf{q} \in S^{3}$ can be represented in a minimal coordinate $\mathbf{a} \in \mathbb{R}^{3}$ where the mapping from $\mathbf{a} \in \mathbb{R}^{3}$ to $\delta \mathbf{q}$ is defined as follows

$$
\delta \mathbf{q}(\mathbf{a})=\frac{1}{2}\left[\frac{\mathbf{a}}{\sqrt{4-\mathbf{a}^{\top} \mathbf{a}}}\right] .
$$

Suppose $\mathbf{q}_{i, I}$ and $\mathbf{q}_{i, I}^{\text {ref }}$ for the $i$-th spacecraft denote true and reference attitude, respectively. Then attitude error $\mathbf{a}_{i, I}$ and angular rate error $\delta \boldsymbol{\omega}_{i, I}$ are defined such that

$$
\begin{aligned}
\delta \mathbf{q}\left(\mathbf{a}_{i, I}\right) & =\mathbf{q}_{i, I} \otimes\left(\mathbf{q}_{i, I}^{\text {ref }}\right)^{-1}, \\
\delta \boldsymbol{\omega}_{i, I} & =\boldsymbol{\omega}_{i, I}-\boldsymbol{\omega}_{i, I}^{\text {ref }} .
\end{aligned}
$$

The kinematic differential equation for the attitude quaternion for the $i$-th spacecraft body frame can be expressed in two equivalent forms

$$
\dot{\mathbf{q}}_{i, I}=\frac{1}{2} \Omega\left(\boldsymbol{\omega}_{i, I}\right) \mathbf{q}_{i, I}=\frac{1}{2} \Theta\left(\mathbf{q}_{i, I}\right) \boldsymbol{\omega}_{i, I}
$$

where $\Omega(\boldsymbol{\omega})$ and $\Theta(\mathbf{q})$ are matrices defined as

$$
\Omega(\boldsymbol{\omega})=\left[\begin{array}{cc}
-\boldsymbol{\omega}^{\times} & \boldsymbol{\omega} \\
-\boldsymbol{\omega}^{\top} & 0
\end{array}\right], \quad \Theta(\mathbf{q})=\left[\begin{array}{c}
q_{s} \mathbf{I}_{3}+\mathbf{q}_{v}^{\times} \\
-\mathbf{q}_{v}^{\top}
\end{array}\right] .
$$

where the superscript $x$ denotes a skew symmetric matrix. The attitude rate of the $i$-th spacecraft is propagated with the following

$$
\dot{\boldsymbol{\omega}}_{i, I}=-\mathbf{J}^{-1} \boldsymbol{\omega}_{i, I} \times \mathbf{J} \boldsymbol{\omega}_{i, I}+\mathbf{J}^{-1} \mathbf{w}_{a}
$$

where $\mathbf{J}$ is the inertia tensor of the spacecraft in the body frame and $\mathbf{w}_{a} \sim$ $\mathcal{N}\left(\mathbf{0}_{3 \times 1}, \mathbf{W}_{a}\right)$ is the attitude process noise modeled as a torque perturbation with zero-mean white Gaussian noise.

\subsection{Review of Error State Estimation}

Because the standard EKF does not strictly enforce the manifold constraint for quaternion, we estimate the error state estimation for the attitude compo-

nents. This is similar to the conventional attitude estimation techniques such as 
the Multiplicative EKF (MEKF) (Markley, 2003). The main idea is to estimate attitude error in a minimal coordinate at each step while using a quaternion to provide a nonsingular attitude representation overall. The filtering involves three steps: time update, measurement update, and reset. This section reviews the time update of the error state and its covariance, as well as the reset step.

The state variables of the $i$-th spacecraft $\mathbf{x}_{i}$ are defined as

$$
\mathbf{x}_{i}=\left[\mathbf{p}_{i, L} ; \quad \mathbf{v}_{i, L} ; \quad \mathbf{q}_{i, I} ; \boldsymbol{\omega}_{i, I}\right]
$$

where $\mathbf{p}_{i, L}$ and $\mathbf{v}_{i, L}$ are relative positions and velocities of the $i$-th spacecraft with respect to the swarm reference LVLH frame. Attitude parameters $\mathbf{q}_{i, I}$ and $\boldsymbol{\omega}_{i, I}$ are the quaternion and the angular rate of the $i$-th spacecraft with respect to the Earth-Centered Inertial (ECI) frame, respectively. Precisely speaking, translational states and rotational states are expressed with respect to different frames (i.e. the LVLH and the ECI frames). This is a convenient choice made to simplify the relative orbital and attitude dynamics models.

The attitude parameters are decomposed into some reference and error $\mathbf{q}_{i, I}=$ $\delta \mathbf{q}\left(\mathbf{a}_{i, I}\right) \otimes \mathbf{q}_{i, I}^{\mathrm{ref}}$ and $\boldsymbol{\omega}_{i, I}=\boldsymbol{\omega}_{i, I}^{\mathrm{ref}}+\delta \boldsymbol{\omega}_{i, I}$, as defined in Eqs. (6) and 7). Nonsingular representation of $\mathbf{x}_{i}$ is denoted by the reference state vector

$$
\mathbf{x}_{i}^{\mathrm{ref}}=\left[\mathbf{p}_{i, L} ; \quad \mathbf{v}_{i, L} ; \quad \mathbf{q}_{i, I}^{\mathrm{ref}} ; \quad \boldsymbol{\omega}_{i, I}^{\mathrm{ref}}\right] .
$$

At each filtering time step, the actual state to be estimated is the minimal coordinate representation of state with respect to $\mathbf{q}_{i, I}^{\text {ref }}$ and $\boldsymbol{\omega}_{i, I}^{\text {ref }}$ defined as

$$
\mathbf{x}_{i}^{\min }=\left[\mathbf{p}_{i, L} ; \quad \mathbf{v}_{i, L} ; \quad \mathbf{a}_{i, I} ; \quad \delta \boldsymbol{\omega}_{i, I}\right] .
$$

We refer to this as the minimal state vector of the $i$-th spacecraft, denoted by the superscript min. At each step, $\mathbf{q}_{i, I}^{\mathrm{ref}}$ and $\boldsymbol{\omega}_{i, I}^{\mathrm{ref}}$ are selected such that the prior estimate of $\mathbf{a}_{i, I}$ and $\delta \boldsymbol{\omega}_{i, I}$ are identically zero.

The state vector $\mathbf{x}_{i}$ resides on a manifold $\mathcal{M}=\mathbb{R}^{6} \times S^{3} \times \mathbb{R}^{3}$ and we can extend the notion of group operator to states in $\mathcal{M}$ as follows. Suppose $\mathbf{x}^{\prime}, \mathbf{x} \in$ $\mathcal{M}$. Then the group operator $\boxplus$ is defined as

$$
\mathbf{x}^{\prime} \boxplus \mathbf{x}=\left[\begin{array}{c}
\mathbf{p}^{\prime}+\mathbf{p} \\
\mathbf{v}^{\prime}+\mathbf{v} \\
\mathbf{q}^{\prime} \otimes \mathbf{q} \\
\boldsymbol{\omega}^{\prime}+\omega
\end{array}\right]
$$

Suppose the error state between two states is defined as $\Delta \mathbf{x}=\mathbf{x}^{\prime} \boxplus \mathbf{x}^{-1} \in \mathcal{M}$,

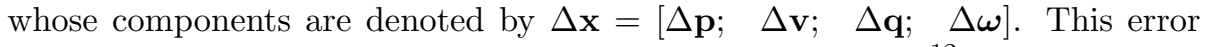
state can be parameterized by a minimal state error $\Delta \chi \in \mathbb{R}^{12}$ defined as

$$
\Delta \chi=[\Delta \mathbf{p} ; \quad \Delta \mathbf{v} ; \mathbf{a}(\Delta \mathbf{q}) ; \quad \Delta \boldsymbol{\omega}] .
$$

Finally two states $\mathbf{x}, \mathbf{x}^{\prime}$ and $\Delta \boldsymbol{\chi}$ are related by

$$
\mathbf{x}^{\prime}=\Delta \mathbf{x}(\Delta \chi) \boxplus \mathbf{x}
$$


where $\Delta \mathbf{x}(\cdot): \mathbb{R}^{12} \rightarrow \mathcal{M}$ is the map from $\Delta \boldsymbol{\chi}$ to $\Delta \mathbf{x}$. The DPE applies Eq. (16) at each reset step to apply the correction $\Delta \boldsymbol{\chi}$, expressed in minimal coordinate, to a prior reference state vector to finally obtain the posterior state vector for each spacecraft.

Next, we derive the equation of motion for attitude error states, which is necessary for computing the covariance time update. Taking the time derivative of the error quaternion given by Eq. (6) and substituting Eq. (8), one can obtain

$$
2 \dot{\delta \mathbf{q}}\left(\mathbf{a}_{i, I}\right)=\left[\begin{array}{c}
\boldsymbol{\omega}_{i, I} \\
0
\end{array}\right] \otimes \delta \mathbf{q}\left(\mathbf{a}_{i, I}\right)-\delta \mathbf{q}\left(\mathbf{a}_{i, I}\right) \otimes\left[\begin{array}{c}
\boldsymbol{\omega}_{i, I}^{\mathrm{ref}} \\
0
\end{array}\right] .
$$

Substituting this into Eq. (5) leads to the equation of motion for attitude error

$$
\dot{\mathbf{a}}_{i, I}=\left(2 \dot{\delta \mathbf{q}}\left(\mathbf{a}_{i, I}\right)\right)_{1: 3}=\frac{1}{2}\left(\left(4-\mathbf{a}_{i, I}^{\top} \mathbf{a}_{i, I}\right)^{\frac{1}{2}} \delta \boldsymbol{\omega}_{i, I}-\left(2 \boldsymbol{\omega}_{i, I}^{\mathrm{ref}}+\delta \boldsymbol{\omega}_{i, I}\right) \times \mathbf{a}_{i, I}\right) .
$$

Similarly, the equation of motion for angular rate error can be derived from Eqs. (7) and (10)

$$
\dot{\delta} \boldsymbol{\omega}_{i, I}=-\mathbf{J}^{-1}\left(\delta \boldsymbol{\omega}_{i, I}^{\times} \mathbf{J}\left(\boldsymbol{\omega}_{i, I}^{\mathrm{ref}}+\delta \boldsymbol{\omega}_{i, I}\right)+\boldsymbol{\omega}_{i, I}^{\mathrm{ref} \times} \mathbf{J} \delta \boldsymbol{\omega}_{i, I}\right)+\mathbf{J}^{-1} \mathbf{w}_{a} .
$$

Eqs. (18) and (19) together represent the attitude error dynamics. The Jacobian of error attitude dynamics in Eqs. (18) and (19) with respect to error attitude variables is given by

$$
\begin{aligned}
\left.\frac{\partial \dot{\mathbf{a}}_{i, I}}{\partial \mathbf{a}_{i, I}}\right|_{\mathbf{a}_{i, I}, \delta \boldsymbol{\omega}_{i, I}=0} & =\left.\left(\frac{-\delta \boldsymbol{\omega}_{i, I}^{\top} \mathbf{a}_{i, I}}{2\left(4-\mathbf{a}_{i, I}^{\top} \mathbf{a}_{i, I}\right)^{\frac{1}{2}}}-\boldsymbol{\omega}_{i, I}^{\mathrm{ref} \times}-\frac{1}{2} \delta \boldsymbol{\omega}_{i, I}^{\times}\right)\right|_{\mathbf{a}_{i, I}, \delta \boldsymbol{\omega}_{i, I}=0}=-\boldsymbol{\omega}_{i, I}^{\mathrm{ref} \times} \\
\left.\frac{\partial \dot{\mathbf{a}}_{i, I}}{\partial \delta \boldsymbol{\omega}_{i, I}}\right|_{\mathbf{a}_{i, I}=0} & =\left.\left(\frac{1}{2}\left(4-\mathbf{a}_{i, I}^{\top} \mathbf{a}_{i, I}\right)^{\frac{1}{2}} \mathbf{I}_{3}+\mathbf{a}_{i, I}^{\times}\right)\right|_{\mathbf{a}_{i, I}=0}=\mathbf{I}_{3} \\
\frac{\partial \delta \boldsymbol{\omega}_{i, I}}{\partial \mathbf{a}_{i, I}} & =\mathbf{0}_{3 \times 3} \\
\left.\frac{\partial \delta \boldsymbol{\omega}_{i, I}}{\partial \delta \boldsymbol{\omega}_{i, I}}\right|_{\delta \boldsymbol{\omega}_{i, I}=0} & =-\left.\mathbf{J}^{-1}\left(\delta \boldsymbol{\omega}_{i, I}^{\times} \mathbf{J}-\left(\mathbf{J}\left(\boldsymbol{\omega}_{i, I}^{\mathrm{ref}}+\delta \boldsymbol{\omega}_{i, I}\right)\right)^{\times}+\boldsymbol{\omega}_{i, I}^{\mathrm{ref} \times} \mathbf{J}\right)\right|_{\delta \boldsymbol{\omega}_{i, I}=0} \\
& =\mathbf{J}^{-1}\left(\left(\mathbf{J} \boldsymbol{\omega}_{i, I}^{\mathrm{ref}}\right)^{\times}-\boldsymbol{\omega}_{i, I}^{\mathrm{ref} \times} \mathbf{J}\right)
\end{aligned}
$$

Finally, covariance can be computed by solving the differential Lyapunov equation

$$
\dot{\mathbf{P}}=\mathbf{A P}+\mathbf{P A}^{\top}+\mathbf{B W B}^{\top}
$$

where $\mathbf{A}$ is Jacobian of propagation of states.

$$
\mathbf{A}=\left[\begin{array}{cc}
\mathbf{A}_{t} & \mathbf{0}_{6 \times 6} \\
\mathbf{0}_{6 \times 6} & \mathbf{A}_{a}
\end{array}\right], \quad \mathbf{B}=\left[\begin{array}{cc}
\mathbf{B}_{t} & \mathbf{0}_{6 \times 3} \\
\mathbf{0}_{6 \times 3} & \mathbf{B}_{a}
\end{array}\right], \quad \mathbf{W}=\left[\begin{array}{cc}
\mathbf{W}_{t} & \mathbf{0}_{3 \times 3} \\
\mathbf{0}_{3 \times 3} & \mathbf{W}_{a}
\end{array}\right]
$$


where $\mathbf{A}_{t}$ and $\mathbf{B}_{t}$ are given by HCW equations in Eq. (2) and $\mathbf{A}_{a}$ and $\mathbf{B}_{a}$ are given by

$$
\mathbf{A}_{a}=\left[\begin{array}{cc}
-\boldsymbol{\omega}_{i, I}^{\mathrm{ref} \times} & \mathbf{I}_{3} \\
\mathbf{0}_{3 \times 3} & \mathbf{J}^{-1}\left(\left(\mathbf{J} \boldsymbol{\omega}_{i, I}^{\mathrm{ref}}\right)^{\times}-\boldsymbol{\omega}_{i, I}^{\mathrm{ref} \times} \mathbf{J}\right)
\end{array}\right], \quad \mathbf{B}_{a}=\left[\begin{array}{c}
\mathbf{0}_{3 \times 3} \\
\mathbf{J}^{-1}
\end{array}\right]
$$

Because the translational and rotational dynamics are decoupled, the state matrix preserves a block diagonal structure.

\section{Decentralized Pose Estimation (DPE) Algorithm}

In this section, we delineate the DPE algorithm. The DPE estimates the poses of a local observable subset of spacecraft in a swarm, given the relative sensing and communication network topologies. First, each spacecraft measures the poses of itself and its neighbors. Each spacecraft then communicates its measurements and the associated measurement noise covariances to its communication neighbors $j \in \mathcal{N}_{i}^{c}$. Based on the available communication and relative sensing networks at the given time, the augmented state vector is modified to add newly detected spacecraft and subtract the spacecraft that became unobservable. Finally, each spacecraft jointly estimates the poses of the local spacecraft. This algorithm is summarized in Algorithm 1 and the following sections explain the steps in detail. A copy of the same algorithm is implemented on each spacecraft. First, we introduce the following definition to clearly define the set of spacecraft to be estimated by the $i$-th spacecraft.

Definition 1. The local observable set $\mathcal{V}_{i} \in \mathcal{V}$ for the $i$-th spacecraft is defined as the union of the sensing neighborhood over the communication neighborhood. That is

$$
\mathcal{V}_{i}:=\bigcup_{j \in \overline{\mathcal{N}}_{i}^{\mathrm{c}}} \overline{\mathcal{N}}_{j}^{\mathrm{s}} .
$$

This is the set of agents detected by the $i$-th spacecraft either via communication or via relative sensing in one communication step. The goal of the DPE is for each spacecraft $i \in \mathcal{V}$ to estimate the state for each detected spacecraft $j \in \mathcal{V}_{i}$. Suppose the cardinality of the local observable set is $N_{i}=\operatorname{card}\left(\mathcal{V}_{i}\right)$. We define the reference augmented state vector $\overline{\mathbf{x}}_{i}^{\text {ref }} \in \mathcal{M}^{N_{i}}$ and the minimal state augmented vector $\overline{\mathbf{x}}_{i}^{\text {min }} \in \mathbb{R}^{12 N_{i}}$ for the $i$-th spacecraft as the column concatenation of all states for $j \in \mathcal{V}_{i}$. That is

$$
\overline{\mathbf{x}}_{i}^{\mathrm{ref}}=\left\|_{j \in \mathcal{V}_{i}} \mathbf{x}_{j}^{\mathrm{ref}}, \quad \overline{\mathbf{x}}_{i}^{\mathrm{min}}:=\right\|_{j \in \mathcal{V}_{i}} \mathbf{x}_{j}^{\mathrm{min}}
$$

where $\mathbf{x}_{j}^{\text {ref }}$ and $\mathbf{x}_{j}^{\min }$ correspond to the full and minimal state for the $j$-th spacecraft as defined in Eq. 11] and 13 . 


\subsection{Absolute and Relative Measurement Models}

Each spacecraft $i \in \mathcal{V}$ is assumed to have an absolute pose measurement $\mathbf{y}_{i}$ with respect to the Earth-Centered Inertial (ECI) frame.

$$
\mathbf{y}_{i}=\mathbf{h}^{\mathrm{a}}\left(\mathbf{x}_{i}, \mathbf{p}_{L, I}, \mathbf{q}_{L, I}\right)+\boldsymbol{\psi}_{i}^{\mathrm{a}}=\left[\begin{array}{c}
R\left(\mathbf{q}_{L, I}\right) \mathbf{p}_{i, L}+\mathbf{p}_{L, I} \\
\mathbf{q}_{i, I}
\end{array}\right]+\boldsymbol{\psi}_{i}^{\mathrm{a}}
$$

where $\boldsymbol{\psi}_{i}^{\text {a denotes measurement noise. }} \mathbf{p}_{L, I}$ and $\mathbf{q}_{L, I}$ describe the LVLH to ECI transformation and are treated as fixed parameters known from the SRFE algorithm. This measurement is available from GPS and a star tracker. We denote the position and the attitude components of this observation as $\mathbf{y}_{i}=\left[\mathbf{p}_{i, I}^{\text {obs }} ; \mathbf{q}_{i, I}^{\text {obs }}\right]$. Since the attitude measurement is given as a quaternion, it is convenient to transform the observation to a pseudo-measurement form (Markley, 2003)

$$
\tilde{\mathbf{y}}_{i}=\left[\begin{array}{c}
R\left(\mathbf{q}_{L, I}\right)\left(\mathbf{p}_{i, I}^{\text {obs }}-\mathbf{p}_{L, I}\right) \\
2\left(\mathbf{q}_{i, I}^{\text {obs }} \otimes\left(\mathbf{q}_{i, I}\right)^{-1}\right)_{1: 3}
\end{array}\right] .
$$

Then this measurement can be modeled as

$$
\tilde{\mathbf{y}}_{i}=\tilde{\mathbf{h}}^{\mathrm{a}}\left(\mathbf{x}_{i}^{\min }\right)+\tilde{\boldsymbol{\psi}}_{i}^{\mathrm{a}}=\left[\begin{array}{c}
\mathbf{p}_{i, L} \\
\mathbf{a}_{i, I}
\end{array}\right]+\tilde{\boldsymbol{\psi}}_{i}^{\mathrm{a}}
$$

where $\tilde{\boldsymbol{\psi}}_{i}^{\mathrm{a}} \sim \mathcal{N}\left(\mathbf{0}_{6 \times}, \tilde{\boldsymbol{\Psi}}_{i}\right)$ is the absolute pseudo-measurement noise vector.

In addition to its absolute measurement, each spacecraft may have relative measurements, possibly multiple at a given time. In this paper, each relative measurement is assumed to be a pose measurement provided by a monocular camera (Garrido-Jurado et al. 2014). The availability of relative measurements depends on the physical constraints of the given sensors such as range, fieldof-view (FOV), and lighting. This information is captured by the edges in the relative sensing graph $\mathcal{G}_{s}$. The relative measurement is assumed to give the relative pose of the observed spacecraft with respect to the observer. Let $\mathbf{y}_{j, i}=\left[\mathbf{p}_{j, i}^{\text {obs }} ; \mathbf{q}_{j, i}^{\text {obs }}\right]$ denote the pose of the $j$-th spacecraft relative to the $i$-th spacecraft. The relative attitude can be written in terms of reference and error attitude as follows

$$
\begin{aligned}
\mathbf{q}_{j, i} & =\mathbf{q}_{j, I} \otimes\left(\mathbf{q}_{i, I}\right)^{-1} \\
& =\delta \mathbf{q}\left(\mathbf{a}_{j, I}\right) \otimes \mathbf{q}_{j, i}^{\mathrm{ref}} \otimes \delta \mathbf{q}\left(-\mathbf{a}_{i, I}\right)
\end{aligned}
$$

where $\mathbf{q}_{j, i}^{\text {ref }}=\mathbf{q}_{j, I}^{\text {ref }} \otimes\left(\mathbf{q}_{i, I}^{\text {ref }}\right)^{-1}$. In the same way as absolute measurement, it is more convenient to transform the relative measurement to a minimal parameterization. We define the relative pseudo measurement by

$$
\tilde{\mathbf{y}}_{j, i}=\left[\begin{array}{c}
\mathbf{p}_{j, i}^{\mathrm{obs}} \\
\mathbf{a}_{j, i}^{\mathrm{obs}}
\end{array}\right]
$$

where $\mathbf{a}_{j, i}^{\text {obs }}=2\left(\mathbf{q}_{j, i}^{\text {obs }} \otimes\left(\mathbf{q}_{j, i}^{\text {ref }}\right)^{-1}\right)_{1: 3}$. Therefore, the minimal relative pose measurement of the $j$-th spacecraft with respect to the $i$-th spacecraft is given by

$$
\tilde{\mathbf{y}}_{j, i}=\tilde{\mathbf{h}}^{\mathrm{r}}\left(\mathbf{x}_{i}^{\min }, \mathbf{x}_{j}^{\min }, \mathbf{x}_{i}^{\mathrm{ref}}\right)+\tilde{\boldsymbol{\psi}}_{j, i}^{\mathrm{r}} .
$$


where $\tilde{\boldsymbol{\psi}}_{j, i}^{\mathrm{r}}$ is relative pseudo-measurement noise and the measurement model is given by

$$
\tilde{\mathbf{h}}^{\mathrm{r}}\left(\mathbf{x}_{i}^{\min }, \mathbf{x}_{j}^{\min }, \mathbf{x}_{i}^{\mathrm{ref}}\right)=\left[\begin{array}{c}
R\left(\delta \mathbf{q}\left(\mathbf{a}_{i, I}\right)\right) R\left(\mathbf{q}_{i, I}^{\mathrm{ref}}\right) R\left(\mathbf{q}_{L, I}\right)^{\top}\left(\mathbf{p}_{j, L}-\mathbf{p}_{i, L}\right) \\
2\left(\delta \mathbf{q}\left(\mathbf{a}_{j, I}\right) \otimes \mathbf{q}_{j, I}^{\mathrm{ref}} \otimes \delta \mathbf{q}\left(-\mathbf{a}_{i, I}\right) \otimes\left(\mathbf{q}_{j, I}^{\mathrm{ref}}\right)^{-1}\right)_{1: 3}
\end{array}\right] .
$$

From this, the Jacobian of relative pseudo-measurement with respect to each minimal state variable can be computed as follows

$$
\begin{aligned}
\frac{\partial \tilde{\mathbf{h}}^{\mathrm{r}}}{\partial \mathbf{p}_{i, L}} & =\left[\begin{array}{c}
-R\left(\mathbf{q}_{i, I}^{\mathrm{ref}}\right) R\left(\mathbf{q}_{L, I}\right)^{\top} \\
\mathbf{0}_{3 \times 3}
\end{array}\right] \\
\frac{\partial \tilde{\mathbf{h}}^{\mathrm{r}}}{\partial \mathbf{p}_{j, L}} & =\left[\begin{array}{c}
R\left(\mathbf{q}_{i, I}^{\mathrm{ref}}\right) R\left(\mathbf{q}_{L, I}\right)^{\top} \\
\mathbf{0}_{3 \times 3}
\end{array}\right] \\
\frac{\partial \tilde{\mathbf{h}}^{\mathrm{r}}}{\partial \mathbf{a}_{i, I}} & =\left[\begin{array}{c}
{\left[R\left(\mathbf{q}_{i, I}^{\mathrm{ref}}\right) R\left(\mathbf{q}_{L, I}\right)^{\top}\left(\mathbf{p}_{j, L}-\mathbf{p}_{i, L}\right)\right]^{\times}} \\
-R\left(\mathbf{q}_{j, i}^{\mathrm{ref}}\right)
\end{array}\right] \\
\frac{\partial \tilde{\mathbf{h}}^{\mathrm{r}}}{\partial \mathbf{a}_{j, I}} & =\left[\begin{array}{c}
\mathbf{0}_{3 \times 3} \\
\mathbf{I}_{3}
\end{array}\right]
\end{aligned}
$$

\subsection{Communication and Augmented Sensing}

At every communication step, each spacecraft broadcasts its sensing information including both the absolute and the relative measurements. Absolute sensing information is defined as $\mathcal{M}_{i}^{\mathrm{a}}=\left(\mathbf{y}_{i}, \mathbf{\Psi}_{i}, i\right)$. For each edge in the relative sensing graph $\mathcal{G}_{s}$, the relative sensing information is defined as $\left(\mathbf{y}_{j, i}, \boldsymbol{\Psi}_{j, i},(i, j)\right)$. Then, we define the set $\mathcal{M}_{i}^{\mathrm{r}}$ to be the set of relative sensing information for all of the direct measurements the $i$-th spacecraft makes:

$$
\mathcal{M}_{i}^{\mathrm{r}}=\left\{\left(\mathbf{y}_{j, i}, \boldsymbol{\Psi}_{j, i},(i, j)\right) \mid j \in \mathcal{N}_{i}^{\mathrm{s}}\right\}
$$

where $\mathcal{N}_{i}^{\mathrm{s}}$ denotes the neighbors of the $i$-th spacecraft in the relative sensing graph $\mathcal{G}^{\mathrm{s}}$. At each communication time step, each spacecraft broadcasts $\mathcal{M}_{i}^{\mathrm{a}}$ and $\mathcal{M}_{i}^{\mathrm{r}}$ to its communication neighbors.

Each spacecraft also collects the information broadcast by its neighbors. The set of all of the relative sensing edges collected by the $i$-th spacecraft is

$$
\overline{\mathcal{E}}_{i}^{\mathrm{s}}:=\left\{(j, k) \in \mathcal{E}^{\mathrm{s}} \mid j \in \overline{\mathcal{N}}_{i}^{\mathrm{c}}\right\}
$$

The augmented relative observation, measurement model, and noise are defined as

$$
\begin{gathered}
\overline{\mathbf{y}}_{i}^{\mathrm{r}}:=\left\|_{(j, k) \in \overline{\mathcal{E}}_{i}^{\mathrm{s}}} \tilde{\mathbf{y}}_{k, j}, \quad \overline{\mathbf{h}}_{i}^{\mathrm{r}}\left(\overline{\mathbf{x}}_{i}^{\min }, \overline{\mathbf{x}}_{i}^{\mathrm{ref}}\right):=\right\|_{(j, k) \in \overline{\mathcal{E}}_{i}} \tilde{\mathbf{h}}^{\mathrm{r}}\left(\mathbf{x}_{j}^{\min }, \mathbf{x}_{k}^{\min }, \mathbf{x}_{j}^{\mathrm{ref}}\right) \\
\overline{\boldsymbol{\psi}}_{i}^{\mathrm{r}}:=\|_{(j, k) \in \overline{\mathcal{E}}_{i}^{\mathrm{s}}} \tilde{\boldsymbol{\psi}}_{k, j}^{\mathrm{r}}
\end{gathered}
$$

which are column concatenations over all of the relative sensing edges available

to the $i$-th spacecraft. Similarly, each spacecraft collects all of the absolute measurements

$$
\overline{\mathbf{y}}_{i}^{\mathrm{a}}:=\left\|_{j \in \overline{\mathcal{N}}_{i}^{\mathrm{c}}} \tilde{\mathbf{y}}_{j}, \quad \overline{\mathbf{h}}_{i}^{\mathrm{a}}\left(\overline{\mathbf{x}}_{i}^{\mathrm{min}}\right):=\right\|_{j \in \overline{\mathcal{N}}_{i}^{\mathrm{c}}} \tilde{\mathbf{h}}^{\mathrm{a}}\left(\mathbf{x}_{j}^{\mathrm{min}}\right), \quad \overline{\boldsymbol{\psi}}_{i}^{\mathrm{a}}:=\|_{j \in \overline{\mathcal{N}}_{i}^{\mathrm{c}}} \tilde{\boldsymbol{\psi}}_{j}^{\mathrm{a}}
$$


The total augmented measurement is the collection of all of the relative and absolute measurements. That is, $\overline{\mathbf{y}}_{i}=\left[\overline{\mathbf{y}}_{i}^{\mathrm{a}} ; \overline{\mathbf{y}}_{i}^{\mathrm{r}}\right], \overline{\mathbf{h}}_{i}=\left[\overline{\mathbf{h}}_{i}^{\mathrm{a}} ; \overline{\mathbf{h}}_{i}^{\mathrm{r}}\right]$, and $\overline{\boldsymbol{\psi}}_{i}=\left[\overline{\boldsymbol{\psi}}_{i}^{\mathrm{a}} ; \overline{\boldsymbol{\psi}}_{i}^{\mathrm{r}}\right]$, such that the augmented measurement equation becomes

$$
\overline{\mathbf{y}}_{i}=\overline{\mathbf{h}}_{i}\left(\overline{\mathbf{x}}_{i}^{\min }, \overline{\mathbf{x}}_{i}^{\mathrm{ref}}\right)+\overline{\boldsymbol{\psi}}_{i} .
$$

The corresponding Jacobian linearized around the estimates $\hat{\overline{\mathbf{x}}}_{i}^{\min }$ and $\hat{\mathbf{x}}_{i}^{\text {ref }}$ becomes

$$
\overline{\mathbf{H}}_{i}=\left.\frac{\partial \overline{\mathbf{h}}_{i}\left(\overline{\mathbf{x}}_{i}^{\min }, \hat{\mathbf{x}}_{i}^{\mathrm{ref}}\right)}{\partial \overline{\mathbf{x}}_{i}^{\min }}\right|_{\overline{\mathbf{x}}_{i}^{\min }=\hat{\mathbf{x}}_{i}^{\min }} .
$$

Since all of the measurement models depend only on one or two spacecraft states at a time, each row of $\overline{\mathbf{H}}_{i}$ will be sparse.

For each spacecraft $i \in \mathcal{V}$, we have the propagation models for the full and the minimal state vectors

$$
\begin{aligned}
\dot{\mathbf{x}}_{i}^{\text {ref }} & =\mathbf{f}\left(\mathbf{x}_{i}^{\text {ref }}\right) \\
\dot{\mathbf{x}}_{i}^{\text {min }} & =\mathbf{f}^{\text {min }}\left(\mathbf{x}_{i}^{\text {min }}, \mathbf{x}_{i}^{\text {ref }}\right)
\end{aligned}
$$

where the reference state model is given by collecting Eqs. (2), (8), and $(10)$ and the minimal propagation model is given by Eqs. (2), (18), and (19). Recall the augmented state for the $i$-th spacecraft is $\overline{\mathbf{x}}_{i}^{\text {ref }}$. Then, the augmented dynamical system for all spacecraft $j \in \mathcal{V}_{i}$ is given by

$$
\begin{aligned}
\dot{\overline{\mathbf{x}}}_{i}^{\mathrm{ref}} & =\overline{\mathbf{f}}_{i}\left(\overline{\mathbf{x}}_{i}^{\mathrm{ref}}\right):=\|_{j \in \mathcal{V}_{i}} \mathbf{f}\left(\mathbf{x}_{j}^{\mathrm{ref}}\right) \\
\dot{\overline{\mathbf{x}}}_{i}^{\min } & =\overline{\mathbf{f}}_{i}^{\min }\left(\overline{\mathbf{x}}_{i}^{\min }, \overline{\mathbf{x}}_{i}^{\mathrm{ref}}\right):=\|_{j \in \mathcal{V}_{i}} \mathbf{f}^{\min }\left(\mathbf{x}_{j}^{\min }, \mathbf{x}_{j}^{\mathrm{ref}}\right) .
\end{aligned}
$$

Integrating Eq. (48) propagates the previous prior estimate $\hat{\mathbf{x}}_{i}^{\text {ref }+}(t-1)$ to the current posterior estimate $\hat{\overline{\mathbf{x}}}_{i}^{\text {ref- }}(t)$, where the superscript - and + denotes prior and posterior, respectively. Eq. (49), instead, is used to define the augmented Jacobian

$$
\overline{\mathbf{A}}_{i}=\frac{\partial \overline{\mathbf{f}}_{i}^{\min }\left(\overline{\mathbf{x}}_{i}^{\min }, \hat{\mathbf{x}}_{i}^{\text {ref }}\right)}{\partial \overline{\mathbf{x}}_{i}^{\min }} \|_{\overline{\mathbf{x}}_{i}^{\min }=\hat{\mathbf{x}}_{i}^{\min }} .
$$

Since the propagation of each state is decoupled, Eq. (50) is a block diagonal where the diagonal block corresponding to $j \in \mathcal{V}_{i}$ is given by $\mathbf{A}\left(\mathbf{x}_{j}^{\text {ref }}\right)$ from Eq. 26). Using these equations, the augmented posterior covariance from the previous step can be updated to the current prior covariance using

$$
\dot{\overline{\mathbf{P}}}_{i}=\overline{\mathbf{A}}_{i} \overline{\mathbf{P}}_{i}+\overline{\mathbf{P}}_{i} \overline{\mathbf{A}}_{i}^{\top}+\overline{\mathbf{B}}_{i} \overline{\mathbf{W}}_{i} \overline{\mathbf{B}}_{i}^{\top} .
$$

While the augmented state matrix $\overline{\mathbf{A}}_{i}$ and the process noise covariance term $\overline{\mathbf{B}}_{i} \overline{\mathbf{W}}_{i} \overline{\mathbf{B}}_{i}^{\top}$ are block diagonal, Eq. 51 has to be solved simultaneously because $\overline{\mathbf{P}}_{i}$ is not diagonal. 
At the measurement update of the DPE, the Kalman gain, the posterior covariance, and the state correction terms are computed similarly to the standard EKF:

$$
\begin{aligned}
\mathbf{K}_{i} & =\overline{\mathbf{P}}_{i}^{-} \overline{\mathbf{H}}_{i}^{\top}\left(\overline{\mathbf{H}}_{i} \overline{\mathbf{P}}_{i}^{-} \overline{\mathbf{H}}_{i}^{\top}+\overline{\mathbf{\Psi}}_{i}\right)^{-1} \\
\overline{\mathbf{P}}_{i}^{+} & =\left(\mathbf{I}-\mathbf{K}_{i} \overline{\mathbf{H}}_{i}\right) \overline{\mathbf{P}}_{i}^{-} \\
\Delta \overline{\boldsymbol{\chi}}_{i} & =\mathbf{K}_{i}\left(\overline{\mathbf{y}}_{i}-\overline{\mathbf{h}}_{i}\left(\overline{\mathbf{x}}_{i}^{\text {min }}, \overline{\mathbf{x}}_{i}^{\mathrm{ref}}\right)\right)
\end{aligned}
$$

Because the correction term $\Delta \bar{\chi}_{i}$ is expressed in the minimal coordinate $\mathbb{R}^{12 N_{i}}$, we apply the reset step to the augmented reference state to recover the posterior estimate of the state

$$
\overline{\mathbf{x}}_{i}^{\mathrm{ref}+}=\Delta \overline{\mathbf{x}}_{i}\left(\Delta \bar{\chi}_{i}\right) \boxplus \overline{\mathbf{x}}_{i}^{\mathrm{ref}^{-}} .
$$

The definition of the group operator $\boxplus$ and the mapping between the tangent space are extended to those for the augmented vector by simply applying the operations for each of $j \in \mathcal{V}_{i}$.

For the purposes of this paper, we implemented the DPE algorithm with specific definitions for the state, measurement, and dynamics models. However, the strategy of defining the augmented state vector and measurements in this paper can be extended to different scenarios.

\subsection{Adding and Subtracting Nodes to Set $\mathcal{V}_{i}$}

The local observable set $\mathcal{V}_{i}$ from Eq. (27) may vary at each time step, based on the relative sensing and communication graphs $\mathcal{G}^{\mathrm{s}}$ and $\mathcal{G}^{\mathrm{c}}$ at the given time. The DPE modifies the augmented state vector $\overline{\mathbf{x}}_{i}^{\text {ref }}$ and its associated covariance $\overline{\mathbf{P}}_{i}$ if the set $\mathcal{V}_{i}$ has changed over time.

A new spacecraft $j$ is added to $\mathcal{V}_{i}$ at time $t$ if a measurement of the new spacecraft becomes available at the new time step. A new measurement becomes available to an agent either when (i) the agent itself or one of its communication neighbors detects a new spacecraft or (ii) the measurement becomes available through the addition of a new communication link. The DPE waits for two consecutive pose measurements, such that the velocities of the new spacecraft are computed by numerical differentiation of the two pose measurements.

The DPE adds the new spacecraft states for $j \in \mathcal{V}_{i}$ to $\hat{\overline{\mathbf{x}}}_{i}$ by adding a new state $\mathbf{x}_{j}^{\text {ref }}$ directly computed from the positions and velocities. A block column and a block row are added to the covariance matrix when initializing the state. Assuming that $\mathbf{x}_{j}^{\text {ref }}$ is independent of $\hat{\mathbf{x}}_{i}$ at the previous time state, the augmented covariance matrix is created by adding a new set of rows and columns with a prescribed specified initial uncertainty. The off-diagonals are zeros since $\mathbf{x}_{j}^{\text {ref }}$ and $\hat{\overline{\mathbf{x}}}_{i}$ are independent.

The observer spacecraft may also stop estimating a spacecraft if a previously estimated spacecraft becomes unobservable. Depending on the application, the dynamics of the unobservable states may be propagated by the dynamics model without the measurement updates for a fixed maximum number of rounds. If a new measurement becomes available for the spacecraft before the maximum 
number of rounds, the measurement update is applied and the count is reset. The spacecraft state and associated covariance blocks are deleted if the count exceeds the specified maximum number.

The DPE algorithms explained in the above sections can be summarized in Algorithm 1

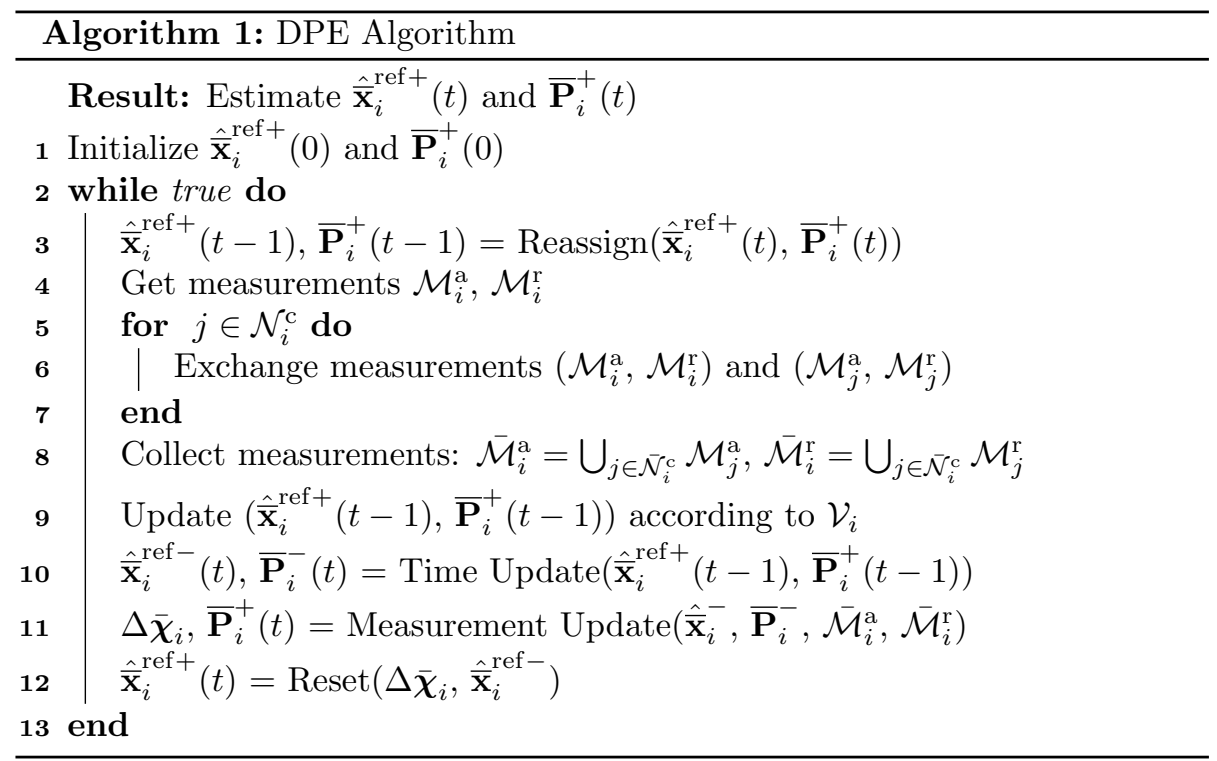

\subsection{Nonlinear Observability}

Assuming that the swarm has limited sensing and limited communication, it is important to determine which subset of spacecraft in the swarm is observable. The observer system for the $i$-th spacecraft in terms of $\overline{\mathbf{x}}_{i}$ is constructed from Eqs. 44 and 48. As usual, the following observability analysis assumes the deterministic nonlinear observer system.

$$
\left\{\begin{aligned}
\dot{\mathbf{x}}_{i} & =\overline{\mathbf{f}}_{i}\left(\overline{\mathbf{x}}_{i}\right) \\
\overline{\mathbf{y}}_{i} & =\overline{\mathbf{h}}_{i}\left(\overline{\mathbf{x}}_{i}\right)
\end{aligned}\right.
$$

We analyze Eq. (56) to determine its nonlinear observability. First, we define the terminology to make the discussion more concrete.

Definition 2. Suppose $i, j \in \mathcal{V}$. We say agent $j$ is observable to agent $i$ if $j \in \mathcal{V}_{i}$ and $\mathbf{x}_{j}$, a subset of state vector $\overline{\mathbf{x}}_{i}$, is observable to $i$.

Definition 3. We say a set of agents $\mathcal{S}_{i} \subseteq \mathcal{V}$ is an observable set with respect to agent $i$ if agent $j$ is observable to agent $i$ for all $j \in \mathcal{S}_{i}$

Recall that $\overline{\mathbf{x}}_{i}=\|_{j \in \mathcal{V}_{i}} \mathbf{x}_{j}$ where $\mathcal{V}_{i} \subseteq \mathcal{V}$. Any agent $j \notin \mathcal{V}_{i}$ is not observable to agent $i$ because it is not a part of the local dynamical system. Therefore $j \in \mathcal{V}_{i}$ is a necessary condition for agent $j$ to be observable to agent $i$. Using this definition, we have the following proposition. 
Proposition 1. Suppose $j \in \overline{\mathcal{N}}_{i}^{\mathrm{c}}$ and $k \in \overline{\mathcal{N}}_{j}^{\mathrm{s}}$ for some $i \in \mathcal{V}$. Then agents $j$ and $k$ are observable to agent $i$.

Proof. We have that $j, k \in \mathcal{V}_{i}$ by Definition 1 , so $\mathbf{x}_{j}$ and $\mathbf{x}_{k}$ are both parts of the state $\overline{\mathbf{x}}_{i}$ estimated by $i$-th agent in the nonlinear system Eq. (56). Now we consider the part of Eq. (56) pertaining to agents $j$ and $k$. We define $\mathbf{w}=$ $\left[\mathbf{x}_{j} ; \mathbf{x}_{k}\right]$.

$$
\left\{\begin{array}{c}
\dot{\mathbf{w}}=\mathbf{f}^{p}(\mathbf{w}) \\
\mathbf{z}=\mathbf{h}^{p}(\mathbf{w})
\end{array}\right.
$$

where $\mathbf{f}^{p}(\mathbf{w})=\left[\mathbf{f}\left(\mathbf{x}_{j}\right) ; \mathbf{f}\left(\mathbf{x}_{k}\right)\right]$ and $\mathbf{h}^{p}(\mathbf{w})=\left[\mathbf{h}^{\mathrm{a}}\left(\mathbf{x}_{j}\right) ; \mathbf{h}^{\mathrm{r}}\left(\mathbf{x}_{j}, \mathbf{x}_{k}\right)\right]$. The measurement model can be written as

$$
\mathbf{h}^{p}(\mathbf{w})=\left[\begin{array}{c}
R\left(\mathbf{q}_{L, I}\right)^{\top} \mathbf{p}_{j, L}+\mathbf{p}_{L, I} \\
\mathbf{q}_{j, I} \\
R\left(\mathbf{q}_{j, I}\right) R\left(\mathbf{q}_{L, I}\right)^{\top}\left(\mathbf{p}_{k, L}-\mathbf{p}_{j, L}\right) \\
\mathbf{q}_{k, I} \otimes\left(\mathbf{q}_{j, I}\right)^{-1}
\end{array}\right]
$$

where $\mathbf{q}_{L, I}$ and $\mathbf{p}_{L, I}$ are known fixed parameters. The zeroth- and first-order Lie derivatives of $\mathbf{h}^{p}$ are given by

$$
\begin{aligned}
\mathfrak{L}^{0} \mathbf{h}^{p}(\mathbf{w}) & =\mathbf{h}^{p}(\mathbf{w}) \\
\mathfrak{L}_{\mathfrak{f}}^{1} \mathbf{h}^{p}(\mathbf{w}) & =\nabla_{\mathbf{w}} \mathbf{h}^{p}(\mathbf{w}) \cdot \mathbf{f}^{p}(\mathbf{w})
\end{aligned}
$$

Based on the Lie derivatives above, the observability matrix is defined as follows

$$
\mathbf{O}=\left\{\nabla_{\mathbf{w}} \mathfrak{L}_{\mathbf{f}^{p}}^{l} \mathbf{h}^{p}(\mathbf{w}) \mid l \in \mathbb{N}\right\}
$$

The observability rank condition (Hermann and Krener, 1977) states that if the observability matrix $\mathbf{O}$ is full column rank, the nonlinear system Eq. (57) is locally weakly observable. One can compute the gradient of the zeroth-order Lie derivative to get

$$
\begin{aligned}
& \nabla_{\mathbf{w}} \mathfrak{L}^{0} \mathbf{h}^{p}(\mathbf{w})=\nabla_{\mathbf{w}} \mathbf{h}^{p}(\mathbf{w}) \\
& =\left[\begin{array}{cccccccc}
R\left(\mathbf{q}_{L, I}\right)^{\top} & \mathbf{0}_{3 \times 3} & \mathbf{0}_{3 \times 4} & \mathbf{0}_{3 \times 3} & \mathbf{0}_{3 \times 3} & \mathbf{0}_{3 \times 3} & \mathbf{0}_{3 \times 4} & \mathbf{0}_{3 \times 3} \\
\mathbf{0}_{4 \times 3} & \mathbf{0}_{4 \times 3} & \mathbf{I}_{4} & \mathbf{0}_{4 \times 3} & \mathbf{0}_{4 \times 3} & \mathbf{0}_{4 \times 3} & \mathbf{0}_{4 \times 4} & \mathbf{0}_{4 \times 3} \\
-R\left(\mathbf{q}_{i, I}\right) R\left(\mathbf{q}_{L, I}\right)^{\top} & \mathbf{0}_{3 \times 3} & \Phi_{1} & \mathbf{0}_{3 \times 3} & R\left(\mathbf{q}_{i, I}\right) R\left(\mathbf{q}_{L, I}\right)^{\top} & \mathbf{0}_{3 \times 3} & \mathbf{0}_{3 \times 4} & \mathbf{0}_{3 \times 3} \\
\mathbf{0}_{4 \times 3} & \mathbf{0}_{4 \times 3} & \Phi_{2} & \mathbf{0}_{4 \times 3} & \mathbf{0}_{4 \times 3} & \mathbf{0}_{4 \times 3} & \Phi_{3} & \mathbf{0}_{4 \times 3}
\end{array}\right]
\end{aligned}
$$

where $\Phi_{1}\left(\mathbf{p}_{j, L}, \mathbf{p}_{k, L}, \mathbf{q}_{j, I}\right)$ and $\Phi_{2}\left(\mathbf{q}_{k, I}\right)$ are some functions that are generally non-zero and $\Phi_{3}=\Phi_{3}\left(\mathbf{q}_{j, I}\right)$ is given by

$$
\Phi_{3}(\mathbf{q})=\left[\begin{array}{cc}
q_{s} \mathbf{I}_{3}+\mathbf{q}_{v}^{\times} & -\mathbf{q}_{v} \\
\mathbf{q}_{v}^{\top} & q_{s}
\end{array}\right]
$$


The gradient of the first-order Lie derivative is

$$
\begin{aligned}
& \nabla_{\mathbf{w}} \mathfrak{L}_{\mathbf{f}^{p}}^{1} \mathbf{h}^{p} \\
& =\left[\begin{array}{cccccccc}
\mathbf{0}_{3 \times 3} & R\left(\mathbf{q}_{L, I}\right)^{\top} & \mathbf{0}_{3 \times 3} & \mathbf{0}_{3 \times 3} & \mathbf{0}_{3 \times 3} & \mathbf{0}_{3 \times 3} & \mathbf{0}_{3 \times 3} & \mathbf{0}_{3 \times 3} \\
\mathbf{0}_{3 \times 3} & \mathbf{0}_{3 \times 3} & * & \frac{1}{2} \Theta\left(\mathbf{q}_{j, I}\right) & \mathbf{0}_{3 \times 3} & \mathbf{0}_{3 \times 3} & \mathbf{0}_{3 \times 3} & \mathbf{0}_{3 \times 3} \\
* & * & * & * & * & R\left(\mathbf{q}_{j, I}\right) R\left(\mathbf{q}_{L, I}\right)^{T} & \mathbf{0}_{3 \times 3} & \mathbf{0}_{3 \times 3} \\
\mathbf{0}_{3 \times 3} & \mathbf{0}_{3 \times 3} & * & * & \mathbf{0}_{3 \times 3} & \mathbf{0}_{3 \times 3} & * & \frac{1}{2} \Phi_{3}\left(\mathbf{q}_{j, I}\right) \Theta\left(\mathbf{q}_{k, I}\right)
\end{array}\right]
\end{aligned}
$$

where an asterisk denotes some non-zero block element of matched dimensions. Because the nonlinear system Eq. (57) is infinitely smooth, $\mathbf{O}$ has an infinite number of rows in general. However, it is sufficient to show that a finite number of rows are linearly independent to determine local weak observability. With this in mind, we consider only the rows corresponding to the zeroth and first Lie derivatives.

$$
\mathbf{O}=\left[\begin{array}{c}
\nabla_{\mathbf{w}} \mathfrak{L}^{0} \mathbf{h}^{p} \\
\nabla_{\mathbf{w}} \mathfrak{L}_{\mathbf{f}^{p}}^{1} \mathbf{h}^{p}
\end{array}\right]
$$

After applying block row elimination, $\mathbf{O}$ reduces to

$\left[\begin{array}{cccccccc}R\left(\mathbf{q}_{L, I}\right)^{\top} & \mathbf{0}_{3 \times 3} & \mathbf{0}_{3 \times 4} & \mathbf{0}_{3 \times 3} & \mathbf{0}_{3 \times 3} & \mathbf{0}_{3 \times 3} & \mathbf{0}_{3 \times 4} & \mathbf{0}_{3 \times 3} \\ \mathbf{0}_{4 \times 3} & \mathbf{0}_{4 \times 3} & \mathbf{I}_{4} & \mathbf{0}_{4 \times 3} & \mathbf{0}_{4 \times 3} & \mathbf{0}_{4 \times 3} & \mathbf{0}_{4 \times 4} & \mathbf{0}_{4 \times 3} \\ \mathbf{0}_{3 \times 3} & \mathbf{0}_{3 \times 3} & \mathbf{0}_{3 \times 4} & \mathbf{0}_{3 \times 3} & R\left(\mathbf{q}_{j, I}\right) R\left(\mathbf{q}_{L, I}\right)^{\top} & \mathbf{0}_{3 \times 3} & \mathbf{0}_{3 \times 4} & \mathbf{0}_{3 \times 3} \\ \mathbf{0}_{4 \times 3} & \mathbf{0}_{4 \times 3} & \mathbf{0}_{4 \times 4} & \mathbf{0}_{4 \times 3} & \mathbf{0}_{4 \times 3} & \mathbf{0}_{4 \times 3} & \Phi_{3}\left(\mathbf{q}_{j, I}\right) & \mathbf{0}_{4 \times 3} \\ \mathbf{0}_{3 \times 3} & R\left(\mathbf{q}_{L, I}\right)^{\top} & \mathbf{0}_{3 \times 4} & \mathbf{0}_{3 \times 3} & \mathbf{0}_{3 \times 3} & \mathbf{0}_{3 \times 3} & \mathbf{0}_{3 \times 4} & \mathbf{0}_{3 \times 3} \\ \mathbf{0}_{4 \times 3} & \mathbf{0}_{4 \times 3} & \mathbf{0}_{4 \times 4} & \frac{1}{2} \Theta\left(\mathbf{q}_{j, I}\right) & \mathbf{0}_{4 \times 3} & \mathbf{0}_{4 \times 3} & \mathbf{0}_{4 \times 4} & \mathbf{0}_{4 \times 3} \\ \mathbf{0}_{3 \times 3} & \mathbf{0}_{3 \times 3} & \mathbf{0}_{3 \times 4} & \mathbf{0}_{3 \times 3} & \mathbf{0}_{3 \times 3} & R\left(\mathbf{q}_{j, I}\right) R\left(\mathbf{q}_{L, I}\right)^{\top} & \mathbf{0}_{3 \times 4} & \mathbf{0}_{3 \times 3} \\ \mathbf{0}_{4 \times 3} & \mathbf{0}_{4 \times 3} & \mathbf{0}_{4 \times 4} & \mathbf{0}_{4 \times 3} & \mathbf{0}_{4 \times 3} & \mathbf{0}_{4 \times 3} & \mathbf{0}_{4 \times 4} & \frac{1}{2} \Phi_{3}\left(\mathbf{q}_{j, I}\right) \Theta\left(\mathbf{q}_{k, I}\right)\end{array}\right]$

Given $\|\mathbf{q}\|=1, R(\mathbf{q}), \Phi_{3}(\mathbf{q})$, and $\Theta(\mathbf{q})$ have full column rank. Therefore $\mathbf{O}$ has full column rank for arbitrary $j \in \overline{\mathcal{N}}_{i}^{\mathrm{c}}$ and $k \in \overline{\mathcal{N}}_{j}^{\mathrm{s}}$. The observability rank condition (Hermann and Krener, 1977) tells that the nonlinear system from Eq. (57) is locally weakly observable.

Finally, we arrive at the following theorem.

Theorem 1. Suppose the detected set of agents $\mathcal{V}_{i} \subseteq \mathcal{V}$ for agent $i$ is defined as in Eq. 27). Then $\mathcal{V}_{i}$ is the largest observable set in $\mathcal{V}$.

Proof. Suppose $j \in \overline{\mathcal{N}}_{i}^{\text {c }}$. Proposition 1 implies that $\overline{\mathcal{N}}_{j}^{\text {s }}$ is an observable set with respect to the agent $i$. Moreover since $\mathcal{V}_{i}$ is defined as the union of all $\overline{\mathcal{N}}_{j}^{\mathrm{s}}$ over $\forall j \in \overline{\mathcal{N}}_{i}^{c}, \mathcal{V}_{i}$ is also an observable set with respect to agent $i$. Recall that $j \in \mathcal{V}_{i}$ is a necessary condition for agent $j$ to be observable to agent $i$ because it has no information on $l \in \mathcal{V} \backslash \mathcal{V}_{i}$. Therefore, we conclude that $j \in \mathcal{V}_{i}$ is a necessary and sufficient condition for agent $j$ to be observable to agent $i$.

Theorem 1 states that all the detected sets of spacecraft $\mathcal{V}_{i}$ are observable in this problem formulation. Moreover, no other spacecraft $j \in \mathcal{V} \backslash \mathcal{V}_{i}$ is observable to the $i$-th spacecraft, given the measurement models and the one-hop 
communication limitation assumed in this problem. In other words, the DPE algorithm estimates the states for all the agents in the largest local observable subset $j \in \mathcal{V}_{i}$.

\section{Consensus Estimation of Swarm Reference Frame}

This section details the Swarm Reference Frame Estimation (SRFE) algorithm. Sophisticated motion planning algorithms typically require a common local reference (e.g. LVLH frame); however, finding such a reference frame is a non-trivial estimation task. All of the spacecraft in the swarm must have an estimate, and the swarm must reach a consensus on the common local reference frame. We apply an information consensus filter (Kamal et al., 2013, Bandyopadhyay and Chung, 2018), which is a decentralized algorithm where a sensor network co-estimates a state vector using the consensus algorithm (Olfati-Saber and Murray, 2004).

In development of the SRFE algorithm, we make the following assumptions:

- the communication graph $\mathcal{G}=\left(\mathcal{V}, \mathcal{E}^{\mathrm{c}}\right)$ is undirected and connected at each time step

- the subset of agents in the swarm has the measurements of the absolute pose of the reference spacecraft

- the degree of the communication graph is upper bounded by a finite bound $d^{\max }$. That is $\operatorname{Card}\left(\mathcal{N}_{i}^{\mathrm{c}}\right)<d^{\max }$ for all $i \in \mathcal{V}$ for some $d^{\max }<+\infty$.

The assumption that the communication graph is undirected may be relaxed so long as the graph is balanced (Olfati-Saber and Murray, 2004, Bandyopadhyay and Chung, 2018). To estimate the common LVLH frame, the state that needs to be estimated is the absolute position and velocity of a reference spacecraft in the ECI frame. Denote $\boldsymbol{\xi}$ to be the reference spacecraft translational state where $\boldsymbol{\xi}=\left[\mathbf{p}_{L, I} ; \mathbf{v}_{L, I}\right]$ and $\mathbf{p}_{L, I}$ and $\mathbf{v}_{L, I}$ are the position and velocity of the reference spacecraft in the ECI. Suppose a subset of spacecraft $\mathcal{W} \subseteq \mathcal{V}$ measures the absolute pose of the reference spacecraft. These absolute pose measurements may be obtained by combining GPS and relative pose measurements, which are assumed to be available for the DPE. Then, the discrete-time dynamics for the whole swarm are given by the following set of equations.

$$
\begin{aligned}
\boldsymbol{\xi}(t+1) & =\mathbf{f}^{s}(\boldsymbol{\xi}(t))+\mathbf{w}^{s}, \quad t=1,2, \ldots \quad \boldsymbol{\xi}(0)=\boldsymbol{\xi}_{0} \\
\boldsymbol{\eta}_{i}(t) & =\mathbf{H}^{s} \boldsymbol{\xi}+\boldsymbol{\psi}_{i}^{s}, \quad i \in \mathcal{W},
\end{aligned}
$$

where $\mathbf{H}^{s}=\left[\begin{array}{ll}\mathbf{I}_{3} & \mathbf{0}_{3 \times 3}\end{array}\right]$ is the absolute measurement model for measurement $\boldsymbol{\eta}_{i}$. Propagation is modeled by a nonlinear function $\mathbf{f}^{s}$. The process and measurement noises are denoted as $\mathbf{w}^{s} \sim \mathcal{N}\left(\mathbf{0}_{6 \times 1}, \mathbf{W}^{s}\right)$ and $\boldsymbol{\psi}_{i}^{s} \sim \mathcal{N}\left(\mathbf{0}_{3 \times 1}, \mathbf{\Psi}^{s}\right)$ respectively and they are assumed to be independent. We define the estimate of $\boldsymbol{\xi}$ by $\hat{\boldsymbol{\xi}}_{i}$ for a spacecraft $i \in \mathcal{V}$. The objective of the SRFE is to estimate $\hat{\boldsymbol{\xi}}_{i}$ in an optimal fashion for all the spacecraft. To this end, we apply the information consensus filter (Kamal et al. 2013) to the distributed dynamical system given by Eqs. 67) and 68). 
The SRFE first computes the proposal information vector $\mathbf{u}_{i}^{0}$ and the proposal information matrix $\mathbf{U}_{i}^{0}$, according to Eqs. (71) and 72 . $N$ denotes the size of the swarm $N=\operatorname{Card}(\mathcal{V})$. This is computed on each spacecraft $i \in \mathcal{V}$ from the prior estimate $\hat{\boldsymbol{\xi}}_{i}^{-}$and the information matrix $\mathbf{J}_{i}^{-}$. Next, the swarm communicates the consensus proposals to its neighbors and iteratively applies consensus $K$ times. For each iteration $k$, the SRFE uses the consensus to compute a posterior information vector and information matrix according to Eqs. (73) and (74) respectively. The consensus coefficient $\epsilon$ must satisfy a stable upper bound $\epsilon<1 / d$ where $d\left(\mathcal{G}^{\mathrm{c}}\right)$ is the maximum degree of the communication graph. By the assumption that the communication graph has a finite degree, we have $d\left(\mathcal{G}^{\mathrm{c}}\right)<d^{\max }$. Then, we choose $\epsilon$ such that $\epsilon<1 / d^{\max }$ to guarantee convergence. The posteriori information state and information matrix are computed according to Eqs. (75) and (76). The algorithm is modified such that it uses nonlinear dynamics for time propagation of state. The Jacobian of $\mathbf{f}^{s}(\boldsymbol{\xi})$ around $\hat{\boldsymbol{\xi}}_{i}$ is defined to be $\mathbf{F}_{i}=\left.\frac{\partial \mathbf{f}^{s}(\boldsymbol{\xi})}{\partial \boldsymbol{\xi}}\right|_{\boldsymbol{\xi}=\hat{\boldsymbol{\xi}}_{i}}$ and this is used for the covariance time propagation. The SRFE algorithm is summarized in Algorithm 2

The SRFE has multiple properties that make it advantageous for the common LVLH estimation. First, the information consensus filter asymptotically approaches the optimal centralized estimate as $K \rightarrow \infty$, assuming the dynamical system is linear (Kamal et al. 2013). The optimal centralized estimate refers to the Kalman filter solution given that the centralized nodes have access to all the measurements Eq. (68), where $\mathbf{f}^{s}$ in Eq. (67) is linear. In practice, it is known that the information consensus filter achieves near-optimal value even if $K$ is small (Kamal et al., 2013). The algorithm is strictly local and decentralized, such that the spacecraft only requires local information exchange. Each of the agents has an estimate of the reference trajectory even if some of the spacecraft do not make a direct measurement. Also, this approach is agnostic to whether the reference spacecraft or target is cooperative or uncooperative, so long as some of the spacecraft in the swarm can measure the absolute position of the reference in the ECI frame. In addition, the LVLH estimation has a unique requirement that the reference trajectory obeys the orbital dynamics. This is because the relative orbital dynamics such as HCW assume that the reference trajectory follows the modeled dynamics. The SRFE algorithm estimates the orbital states of an actual orbiting body; therefore, an unbiased estimate of $\boldsymbol{\xi}$ will also satisfy the orbital dynamics.

\section{Validation of DPE and SRFE by Numerical Simulations}

In the following three subsections, we discuss how the performance of the DPE and the SRFE was verified. Using a satellite inspection mission scenario as an example, we first illustrate the estimator convergence. Second, we quantitatively compare the computational time and the estimation error for an increasing number of spacecraft in the swarm. Finally, the DPE was implemented in a robotic experiment using Caltech's robotic spacecraft simulators called the 


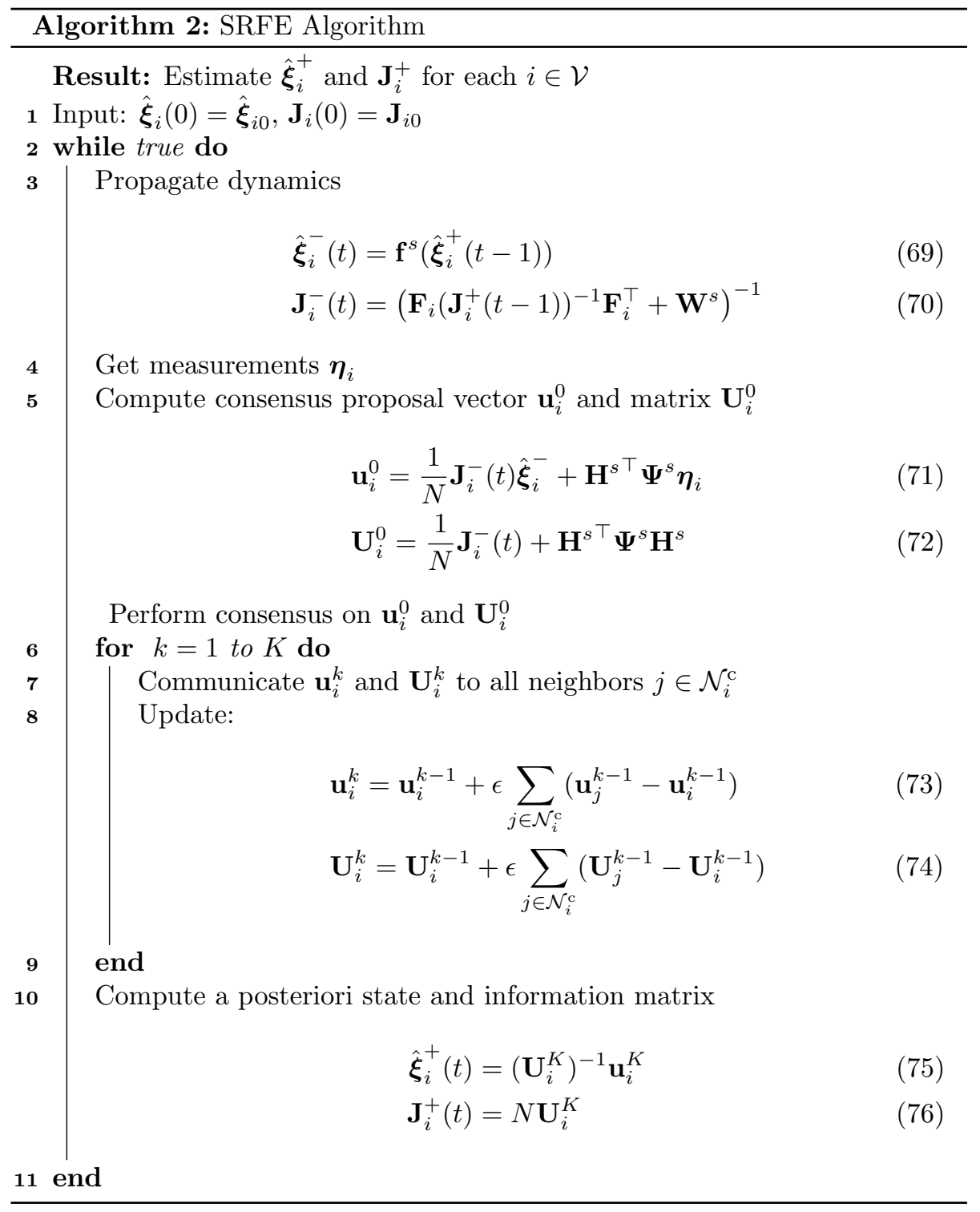

Multi-spacecraft Testbed for Autonomy Research (M-STAR). The experiment considers time-varying relative sensing and communication graphs.

\subsection{Numerical Simulation Example}

This section verifies the performance of the DPE algorithm in a 6DOF numerical simulation example. We consider an example mission scenario in a circular, Low Earth Orbit (LEO) where three spacecraft cooperatively inspect 
one target spacecraft, such as a defunct satellite. The target is uncooperative in the sense that it does not communicate any information with the other spacecraft in the swarm. The three inspector spacecraft are placed in periodic, thrust-free relative spacecraft trajectories referred to as Passive Relative Orbits (PROs) (Scharf et al. 2003) such that the centers of the PROs coincide with the target. Each spacecraft has an elliptical relative orbit with radii of $10 \times 20$ meters. All the spacecraft are initialized in the same orbital plane. The ground truth dynamics of each spacecraft were modeled using (nonlinear) Keplerian dynamics with no perturbations. The DPE uses the linearized HCW dynamics as the propagation model for the relative dynamics and the SRFE integrates the Keplerian dynamics to propagate the target state. For the attitude motion, the spacecraft follow a constant slew rate matching the negative of the mean motion, such that the attitude makes one rotation with one orbit. The absolute sensing uncertainties are selected to be 5 meters in position and 1 degree in attitude. The relative measurement uncertainties are 0.1 meters in position and 0.1 degrees in attitude. The simulation is run for one orbit. We assume that each spacecraft has the relative measurements of the target and the other spacecraft, and that all of the inspecting spacecraft communicate with each other. The sensing and communication graphs are fixed in this simulation example; the dynamic graphs are considered in the robotic experiment. The relative sensing and communication graphs are shown in Figure 2 .

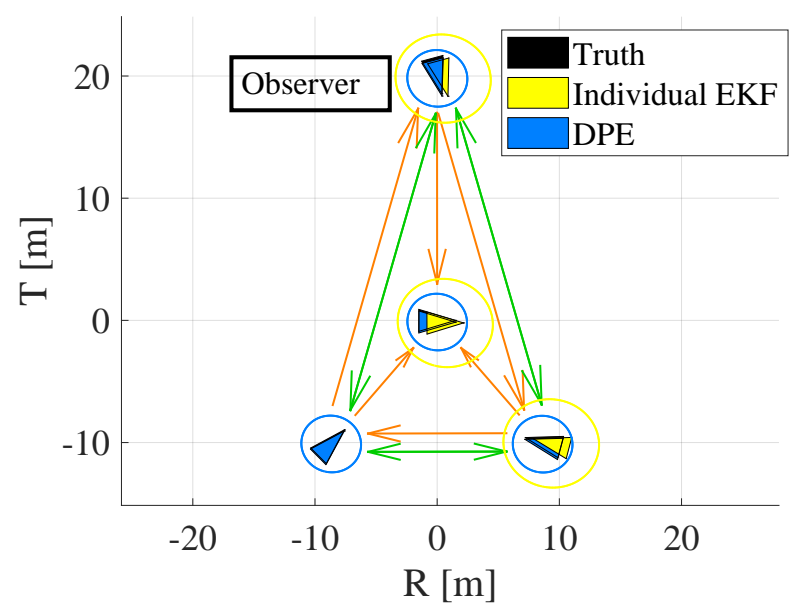

Figure 2: DPE and Individual EKF pose estimates and relative sensing and communication graphs

The inspector spacecraft first uses the SRFE to estimate the target spacecraft orbit state in the ECI frame. Since the target is uncooperative, the team of inspector spacecraft uses the SRFE to collectively estimate the target trajectory, which is then used to define the LVLH frame. Each inspector spacecraft uses its absolute measurement and the relative measurement of the target to create 
a pseudo-measurement of the target absolute position. The maximum degree of the communication graph is $d=2$, so the consensus coefficient is selected to be $\epsilon=0.49$. Each spacecraft uses the target estimate from the SRFE to define the LVLH frame. Next, the DPE estimates the formation pose with respect to the common LVLH frame. The absolute pose measurements are transformed from the ECI frame to the LVLH frame.

As a point of comparison, we also implement the Individual EKF where each spacecraft estimates poses only using its own absolute and relative measurements. For the estimation parameters such as measurement and process noise, the same parameters were used for the Individual EKF as those of the DPE. This represents the case where there is no communication between the spacecraft.

Figure 2 shows the formation pose estimate obtained in the DPE after one orbit. The triangles represent the poses of the spacecraft in the swarm: black for ground truth, blue for the DPE estimate, and yellow for the Individual EKF estimate. The corresponding error ellipse represents the 99.7 percent confidence of the respective position estimates. Figure 2 shows that the position error covariance for the DPE is much smaller than that of the Individual EKF. The local relative sensing graph of the DPE can be viewed as a pose graph. In this example, the DPE can estimate the states for all of the spacecraft in the swarm even though there are only two direct relative measurements available to each spacecraft.

In order to compare the Individual EKF and the DPE, the error metric was defined as the Euclidean norm of the position estimation error: $\| \hat{\mathbf{p}}_{i, L}-$ $\mathbf{p}_{i, L} \|$. Figure $3(\mathrm{a})$ shows the time history of the position estimation error for an inspector spacecraft estimated by another inspector spacecraft. The position error for the DPE is smaller compared to that of the Individual EKF at a steady state. Figure 3(a) also shows that the estimate converges quickly.

Figure 3(b) shows the performance of the SRFE algorithm for each of the three inspector spacecraft. The figure plots the error metric $\left\|\hat{\mathbf{p}}_{L, I}-\mathbf{p}_{L, I}\right\|$ which is the norm of the position estimation error of the reference spacecraft with respect to the inertial frame. The figure shows that the SRFE estimates quickly converge to the true trajectory for all the spacecraft. The high correlation between the three estimate errors is due to the fact that the three estimates converge to a commonly agreed estimate.

\subsection{Scalability Analysis}

We verify the scalability of the DPE in numerical simulations for an increasing number of spacecraft in the swarm. The swarm sizes considered are 5, 100, 150, 200, 250, and 300 spacecraft. For each simulation, we study the estimation accuracy and the computational time of the DPE on each spacecraft. The performance of the DPE is compared against two other filters: the Centralized EKF, which has access to all of the measurements by all of the spacecraft, and the Individual EKF, where each individual spacecraft estimates the states only using its own measurements. The same parameters as those of the DPE were used for the Individual EKF and the Centralized EKF, except for the assumption 


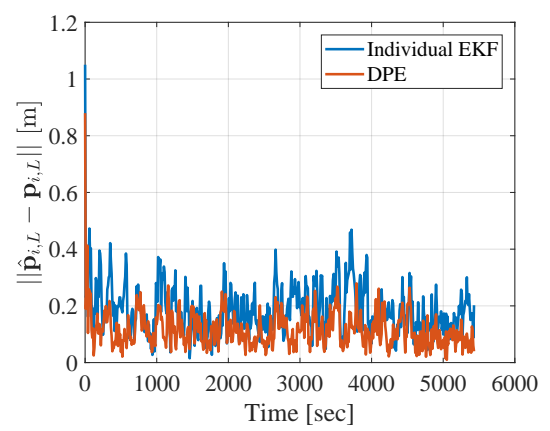

(a) Individual EKF and DPE position estimation error

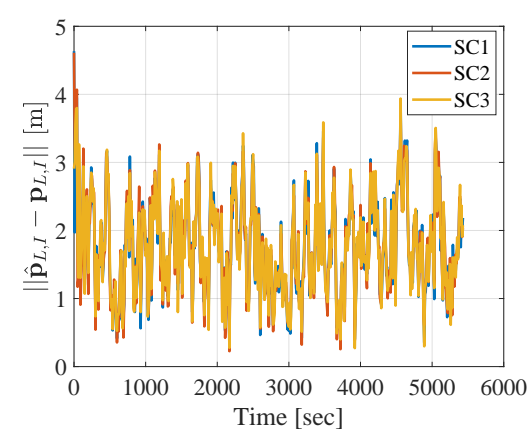

(b) SRFE reference position estimate

Figure 3: Example estimation errors for the DPE and the SRFE algorithms

on the communication. This scalability analysis does not include the computational time of obtaining the measurements. Because the scalability of the SRFE algorithm is established in prior work (Kamal et al. 2013; Bandyopadhyay and Chung, 2018), we assume the absolute reference state of the SRFE is given for this section only.

Given the relative positions, the relative velocities are selected such that all of the spacecraft have a concentric PRO. The relative measurements are obtained as described by Eq. (34). The simulation is run for 3,000 sec, which is approximately half of an orbit.

The relative positions of spacecraft are initialized randomly. We specify a minimum separation distance between spacecraft. The spatial density of the swarm is kept constant for a varying number of spacecraft. The graph edges between spacecraft are created if the Euclidean distance between each pair of spacecraft is below the detection threshold. The relative sensing and communication networks are given by the same graph. We enforce a maximum degree on each agent by pruning edges off the nodes with too many edges. A limited degree physically corresponds to each spacecraft having a restricted number of communication links. With this assumption, the DPE has a bounded number of elements in the augmented state vector regardless of the total number of spacecraft in the swarm. Moreover, we ensure that the communication graph is fully connected in all of the scenarios considered. Figures 4(a) through 4(c) show the example graphs for 5, 100 and 300 spacecraft.

The performance of the DPE is compared against the Centralized and Individual EKFs. The Centralized EKF is a global observer who has access to all of the measurements available in the swarm. While the Centralized EKF is prohibitive for large formations in terms of communication and computation, it quantifies an achievable estimator performance should there be a global estimator. The performance of the DPE, the Centralized EKF, and the Individual EKF are compared in Figures 5(a) and 5(c)] Figure 5(a) shows the position 


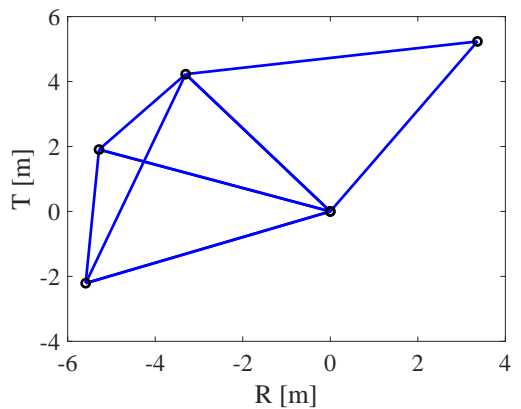

(a) 5 spacecraft

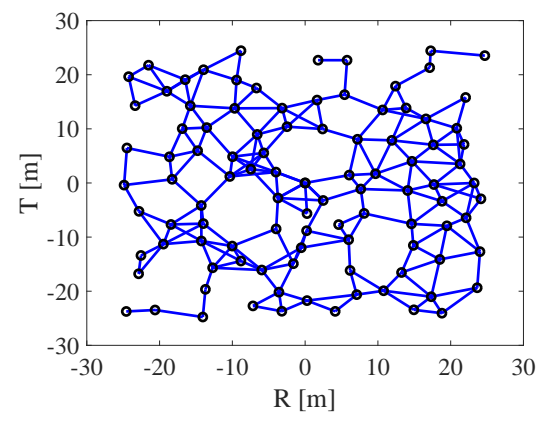

(b) 100 spacecraft

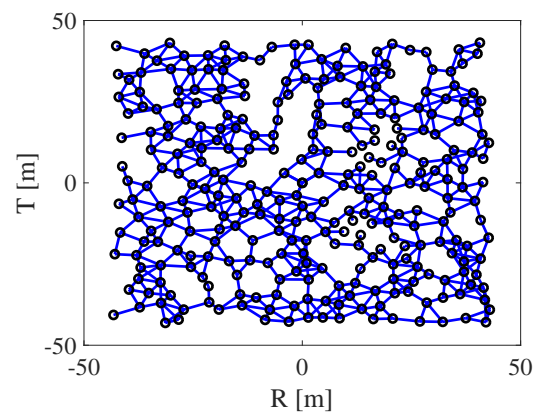

(c) 300 spacecraft

Figure 4: Spacecraft swarm with (a) 5, (b) 100, (c) 300 spacecraft in a common LVLH frame

estimation error standard deviation for different numbers of spacecraft in the swarm. The position estimation error, computed once the steady state has been achieved, is the time-averaged and swarm-averaged absolute position estimate standard deviation. The figure shows that the position estimation error has improved compared to the Individual EKF case. The estimation error of the Centralized EKF is better than that of the DPE, as expected. Because the DPE is a local algorithm, increasing the swarm size does not improve the estimation performance. While the Centralized EKF estimation error improves as the swarm size increase, it has diminishing returns. The incremental information gain due to an additional spacecraft is diluted by uncertainties accumulated over the relative sensing graph hops. The DPE estimate strikes a balance by capturing most of the local information in the swarm while controlling the complexity of the on-board algorithm.

Figure $5(\mathrm{~b})$ shows the computation time required by each spacecraft for the DPE, the Centralized EKF, and the Individual EKF. For the DPE and the Individual EKF, the computation time is averaged over all of the spacecraft in the swarm. The results show that the computation time for the DPE remains constant as the number of spacecraft in the swarm increases. This is expected 
as the DPE is a local algorithm and the graphs have a fixed degree so the size of the state to be estimated is bounded. Figure $5(\mathrm{c})$ compares the number of spacecraft estimated by each estimation algorithm. For the DPE, this is the number of spacecraft included in the local relative sensing graph. For the Individual EKF, this is the size of the relative sensing neighborhood. The bars represent the minimum and maximum number among all of the spacecraft in the swarm. Note that the DPE has a significantly larger (but bounded) number of spacecraft observed by each spacecraft. On average, the number of estimated spacecraft increases by more than a factor of two. The maximum number of spacecraft observed is high for the DPE. This occurs at the part of the swarm where many spacecraft are close to each other. In this example, we restricted the communication and relative sensing graphs to have a maximum degree of $d^{\max }=6$. This ensures that the number of nodes in the local relative sensing graph is bounded. This is also empirically confirmed by the fact that the maximum size of the local sensing graph does not grow as the overall swarm size increases. Note that this maximum size of the local sensing graph can be reduced by choosing a smaller upper bound for the maximum degree of the graph. Also, the simulation did not include the time required to obtain the pose measurements. While the computational complexity for vision-based pose extraction likely has a larger constant than the DPE algorithm, it is also constant with respect to the swarm size.

\section{Experimental Validation Using Spacecraft Simulators}

The DPE algorithm was implemented on-board Caltech's robotic spacecraft simulators, the M-STAR. In the experiment, each spacecraft obtained relative pose measurements using a monocular camera and a computer vision algorithm. This experiment tested the real-time performance of the DPE with time-varying graphs, where the relative sensing graph changed depending on which spacecraft were in the camera's field-of-view (FOV). Because the experiment was constrained to planar motions, the DPE algorithm formulation was modified from the 6-DOF to its 3-DOF analog. With the 3-DOF formulation, the state for the $i$-th spacecraft is selected to be $\mathbf{x}_{i}=\left[\mathbf{p}_{i, L} ; \mathbf{v}_{i, L} ; \theta_{i, L} ; \omega_{i, L}\right]$ where $\mathbf{p}_{i, L}$ and $\mathbf{v}_{i, L}$ denote $2 \mathrm{D}$ position and velocity vectors with respect to the LVLH frame and $\theta_{i, L}$ and $\omega_{i, L}$ denote the $1 \mathrm{D}$ attitude and rotation rate of the spacecraft. The time-varying communication graph was simulated by masking part of the available communication packages.

\subsection{Experimental Setup}

Each spacecraft simulator used air-bearing and on-board air-based thrusters to simulate frictionless dynamics similar to those in space. We used the 3-DOF configuration where the simulators translate and rotate only in a planar motion. Each spacecraft was equipped with a Jetson TX2 computer, a monocular camera with a high FOV lens, and the ArUco visual markers (Garrido-Jurado et al. 2014) on each side as seen in Figure 6. Example images of the detected markers are shown in Figure 7. 


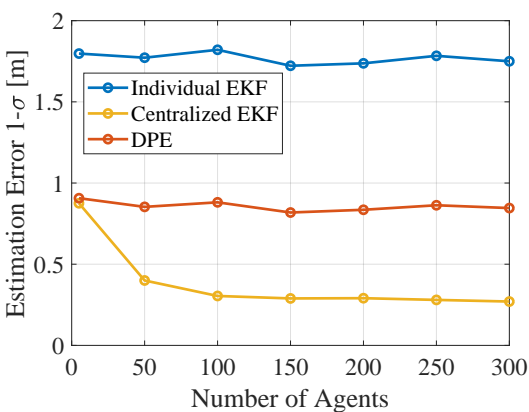

(a) Position estimation accuracy

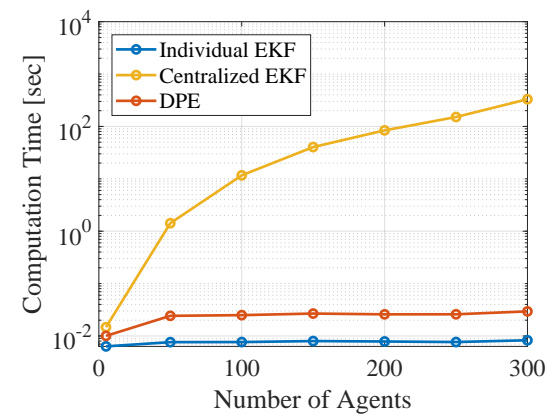

(b) Computation time

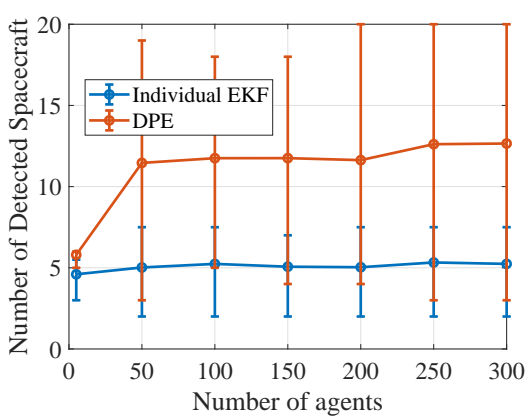

(c) Number of spacecraft estimated

Figure 5: Performance comparison between Individual EKF, Centralized EKF, and DPE

Each spacecraft simulator used a thruster-based controller to follow a prescribed HCW trajectory using the ground truth pose from the motion capture system. We implemented the same formation as the numerical simulation case, where three inspector spacecraft (labeled 1 through 3) orbited around an uncooperative target spacecraft (labeled 4). The attitude dynamics again assume that the spacecraft rotate at the rate of the negative of the mean motion. The resulting trajectories were such that each spacecraft measured at least two spacecraft persistently throughout the trajectory. The time of each orbit was scaled to $262 \mathrm{sec}$, short enough so that the experiment would be completed without depleting compressed-air for the thrusters used to follow the trajectory.

The images from the monocular camera were processed on-board each spacecraft using a standard computer vision algorithm (Garrido-Jurado et al., 2014) to detect the ArUco markers and estimate their full pose. While the ArUcobased algorithm does not address some of the relative pose estimation challenges that result from using electro-optical sensors in a space environment (Opromolla et al. 2017), there exist various other vision-based relative pose estimation al- 


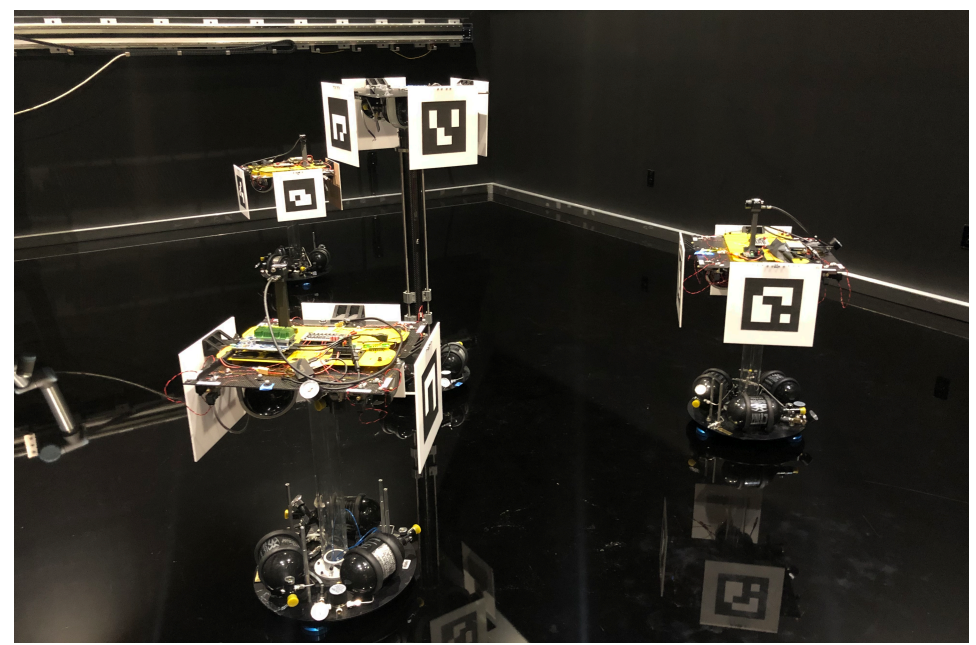

Figure 6: M-STAR with the ArUco markers on the flat epoxy floor
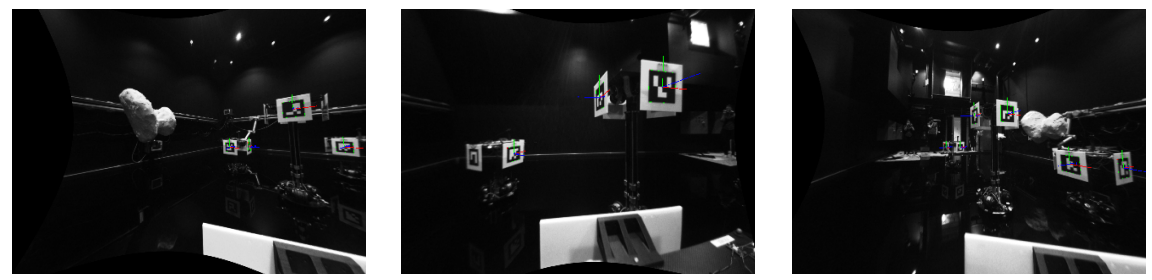

Figure 7: Detected markers as seen from the cameras on-board the spacecraft simulators

gorithms (Kelsey et al., 2006; Capuano et al., 2020; Cassinis et al., 2019) implementing application-specific solution strategies. Because the contribution of the DPE is the decentralized architecture for abstract communication and sensing networks, the DPE can be used in conjunction with most vision-based algorithms available. For this reason, we simplified the experiments by using the ArUco markers and focus on the DPE aspects. The absolute pose measurement $\left[\mathbf{p}_{i, L} ; \theta_{i, L}\right]$ is given from a motion capture system. The motion capture system is typically used to provide the ground truth position of the spacecraft to a sub-millimeter level accuracy; therefore, additional noise was artificially added to make the absolute measurement more realistic. The noise was modeled as zero-mean Gaussian with 0.2 meters standard deviation in translation and 2 degrees standard deviation in rotation.

The on-board computers sent and received information over a wireless network to simulate the inter-spacecraft communication. In order to test the DPE's performance under a time-varying communication graph, some shared information was artificially masked to simulate time-varying inter-satellite communication links. Specifically, we prescribed the edges of the undirected communication 
Table 1: Parameters specified in the DPE

\begin{tabular}{ll}
\hline \hline Parameters & Values \\
\hline \hline Process noise std dev & \\
$\quad$ Translation & $0.03 \mathrm{~m}, 0.01 \mathrm{~m} / \mathrm{sec}$ \\
Attitude & $0.2 \mathrm{deg}, 0.05 \mathrm{deg} / \mathrm{sec}$ \\
\hline Absolute measurement std dev & \\
$\quad$ Translation & $0.2 \mathrm{~m}$ \\
$\quad$ Attitude & $2 \mathrm{deg}$ \\
\hline Relative measurement std dev & \\
Translation & $0.1 \mathrm{~m}$ \\
$\quad$ Attitude & $10 \mathrm{deg}$ \\
\hline Initial uncertainty std dev & \\
$\quad$ Translation & $2 \mathrm{~m}, 0.03 \mathrm{~m} / \mathrm{sec}$ \\
$\quad$ Attitude & $15 \mathrm{deg}, 2 \mathrm{deg} / \mathrm{sec}$ \\
\hline Control interval & $1 \mathrm{sec}$ \\
\hline
\end{tabular}

graph to be

$$
\mathcal{E}^{\mathrm{c}}= \begin{cases}\emptyset, & \text { if } t<20 \mathrm{sec} \\ \{(1,2),(1,3)\}, & \text { if } 20 \mathrm{sec} \leq t<50 \mathrm{sec} \\ \{(1,2),(1,3),(2,3)\}, & \text { if } 50 \mathrm{sec} \leq t\end{cases}
$$

The measurement graph was also allowed to vary between time steps, depending on whether a neighbor spacecraft was visible in the FOV or not.

Table1 1 includes information about the parameters used by the DPE in the experiment. The relative measurement covariance is scaled with the squared Euclidean distance between spacecraft centers to model a larger uncertainty for relative measurement at a large separation distance.

\subsection{Software Architecture}

The Robot Operating System (ROS) was used for interfacing with the sensors and communicating measurements across robots. A block diagram of the software architecture can be seen in Figure 8. After obtaining the pose of the ArUco markers in the FOV using computer vision, each marker pose was transformed into the corresponding spacecraft body frame. This frame transformation was estimated by an extrinsic calibration procedure described in Section 6.3. The relative and absolute measurements and their covariances were then communicated to the prescribed neighbors over the wireless network. When the set of observable agents changed due to time-varying measurement or communication graphs, the method described in Section 3.3 was used to modify the augmented states and covariances. 


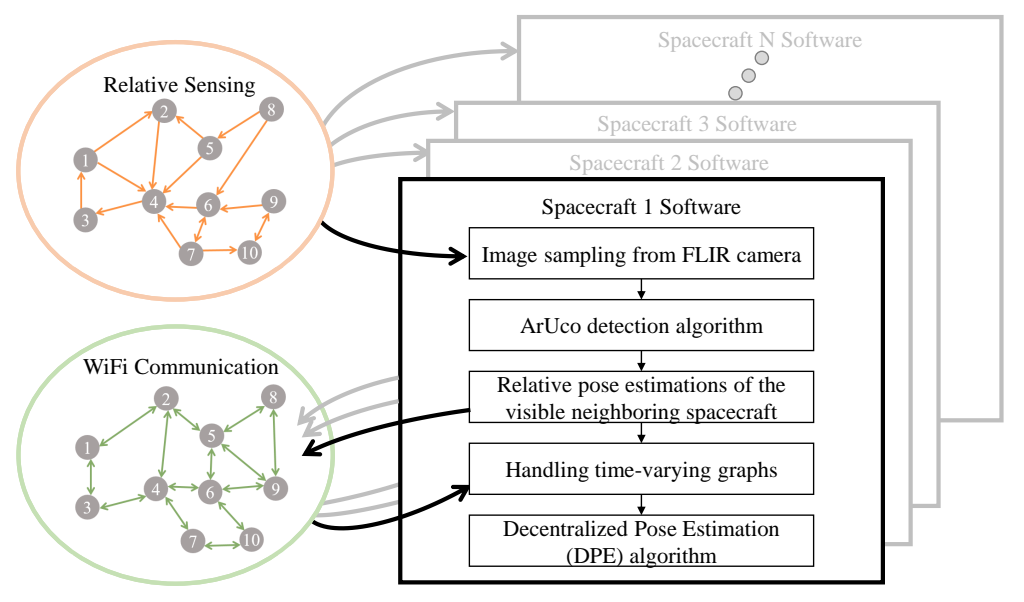

Figure 8: DPE software architecture

\subsection{Camera Calibration}

We performed camera intrinsic and extrinsic calibrations for each pairing of a monocular camera and a spacecraft simulator. For the intrinsic calibration, a pinhole camera model with 6 radial distortion coefficients, 2 tangential distortion coefficients, and 4 thin prism distortion coefficients was used to model the high FOV lens camera. A checkerboard pattern was used as a visual target while the motion capture system collected the camera and the target poses.

Extrinsic calibration was performed to determine the relative pose between the camera frame and the spacecraft body frame. The camera was rigidly attached to the spacecraft body. The camera frame was defined such that its origin coincided with the camera optical center. To perform this calibration, we first used a monocular camera and a computer vision algorithm to extract the relative pose of the ArUco targets. Sufficient pose measurements were collected while the ArUco target was moved around the workspace. At each time step, the relative pose between the spacecraft and the ArUco target was also obtained using the motion capture system. We solved a least-square optimization to retrieve the camera to spacecraft body calibration.

For each relative sensing edge, the relative pose measurement error was computed as detected pose minus the ground truth from the motion capture system. After camera calibration, the standard deviation of relative measurement error was $3.0 \mathrm{~cm}$ along the line of sight, $2.3 \mathrm{~cm}$ in the in-plane perpendicular direction, and $2.9 \mathrm{deg}$ for attitude.

Table 1 includes information about the parameters used by the DPE in the experiment. The relative measurement covariance is scaled with the squared Euclidean distance between spacecraft centers to model a larger uncertainty for relative measurement at a large separation distance. 


\subsection{Experimental Results}

The three inspector spacecraft, shown in Figure 6, ran the DPE algorithm in synchronized rounds once every $1 \mathrm{sec}$, of which the ArUco detection took approximately $0.1 \mathrm{sec}$. Measurement collection and communication were allotted a $0.3 \mathrm{sec}$ period. After the measurement was collected, the DPE step took approximately $10^{-3}$ sec. Figure 9 shows the varying sensing and communication network used by Spacecraft 2 for estimation in the experiments. The orange edges correspond to the relative sensing graph, while the green edges correspond to the communication graph. Initially, there is no communication between any of the spacecraft, and Spacecraft 2 only has access to the measurements it collects: relative measurements obtained using ArUco pose estimation and a measurement of its own absolute pose. At the time $t=20 \mathrm{sec}$, Spacecraft 2 begins to communicate with Spacecraft 1. This allows Spacecraft 2 to have additional relative and absolute measurements. At the time $t=50 \mathrm{sec}$, Spacecraft 2 starts communicating with Spacecraft 3, adding more measurements to its graph. Figure 10 shows the estimation error as a function of time during the experiment. A 2- $\sigma$ uncertainty envelope is plotted around the error, using the estimated covariance matrix. The dashed vertical lines indicate that new communication links are introduced at $t=20 \mathrm{sec}$ and $t=50 \mathrm{sec}$. For every additional communication link, the covariance size decreases, confirming that added relative and absolute measurements provided by communication reduce uncertainty.

Figure 10 also shows that even when the communication graph remains unchanged, the measurement graph also changes due to some spacecraft entering and exiting the camera FOV. Time-varying relative sensing topologies are representative of realistic sensing constraints for a spacecraft swarm. For instance, at $t=37 \mathrm{sec}$ an edge is lost and then regained at $t=40 \mathrm{sec}$. Figure 10 shows that there is a temporary increase in the uncertainty during this period, followed by a decrease when the measurement is restored. This observation supports that additional relative measurements generally help the DPE reduce the estimate uncertainty. There are some other events where relative sensing edges are added and lost (after the second communication link is established at $t=50 \mathrm{sec}$ ), but the covariance did not change noticeably. This is likely explained by the added measurements available to Spacecraft 2 after the communication links to both Spacecraft 1 and 3 are established. These extra measurements provide more observation paths from Spacecraft 1 to Spacecraft 4, adding redundancy so that the loss of a single measurement is not as impactful. These redundant measurements from communication are another advantage of cooperative estimation using the DPE. 

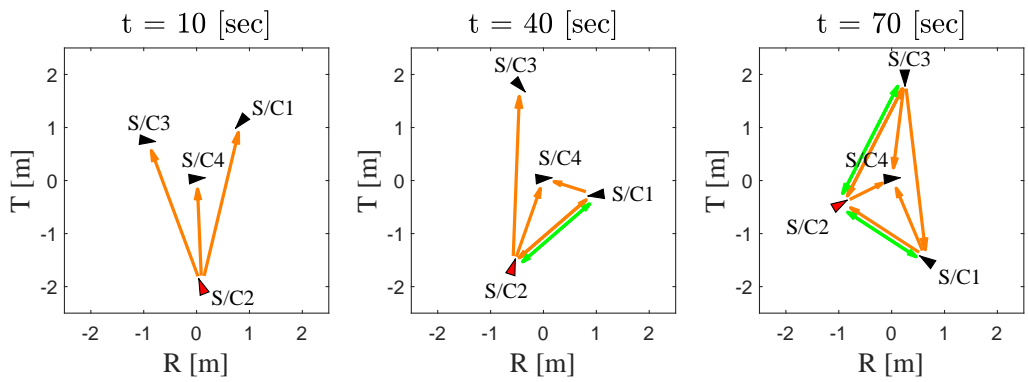

Figure 9: The relative sensing and communication edges as seen by the observer (Spacecraft 2) at different times during the experiment

\section{Conclusion}

We present the Decentralized Pose Estimation (DPE) algorithm that solves the swarm localization problem for formation flying spacecraft. The DPE considers ad hoc relative sensing and communication networks to determine a set of observable spacecraft and shares these spacecraft's measurements to jointly estimate their poses with respect to the LVLH frame at each time step. As a part of the DPE, the Swarm Reference Frame Estimation (SRFE) algorithm applies the information consensus filter to estimate the common LVLH frame in a decentralized fashion. The DPE is a local, decentralized algorithm that has a constant complexity with respect to the swarm size. Numerical simulations verify that the estimation errors of the DPE are improved compared to those for no cooperation cases and that the computation time remains constant as the swarm size increases. An experimental result using the air-bearing spacecraft simulators demonstrates good DPE performance using vision-based relative pose measurements with ad hoc networks.

\section{Acknowledgement}

This research was supported in part by the Jet Propulsion Laboratory, California Institute of Technology, under a contract with the National Aeronautics and Space Administration. The work of Kai Matsuka was supported by the National Science Foundation Graduate Research Fellowship under Grant No. DGE 1745301. We also would like to thank Alexei Harvard for his help on camera calibration as well as to Jennifer Sun and Amir Rahmani for their technical support. 

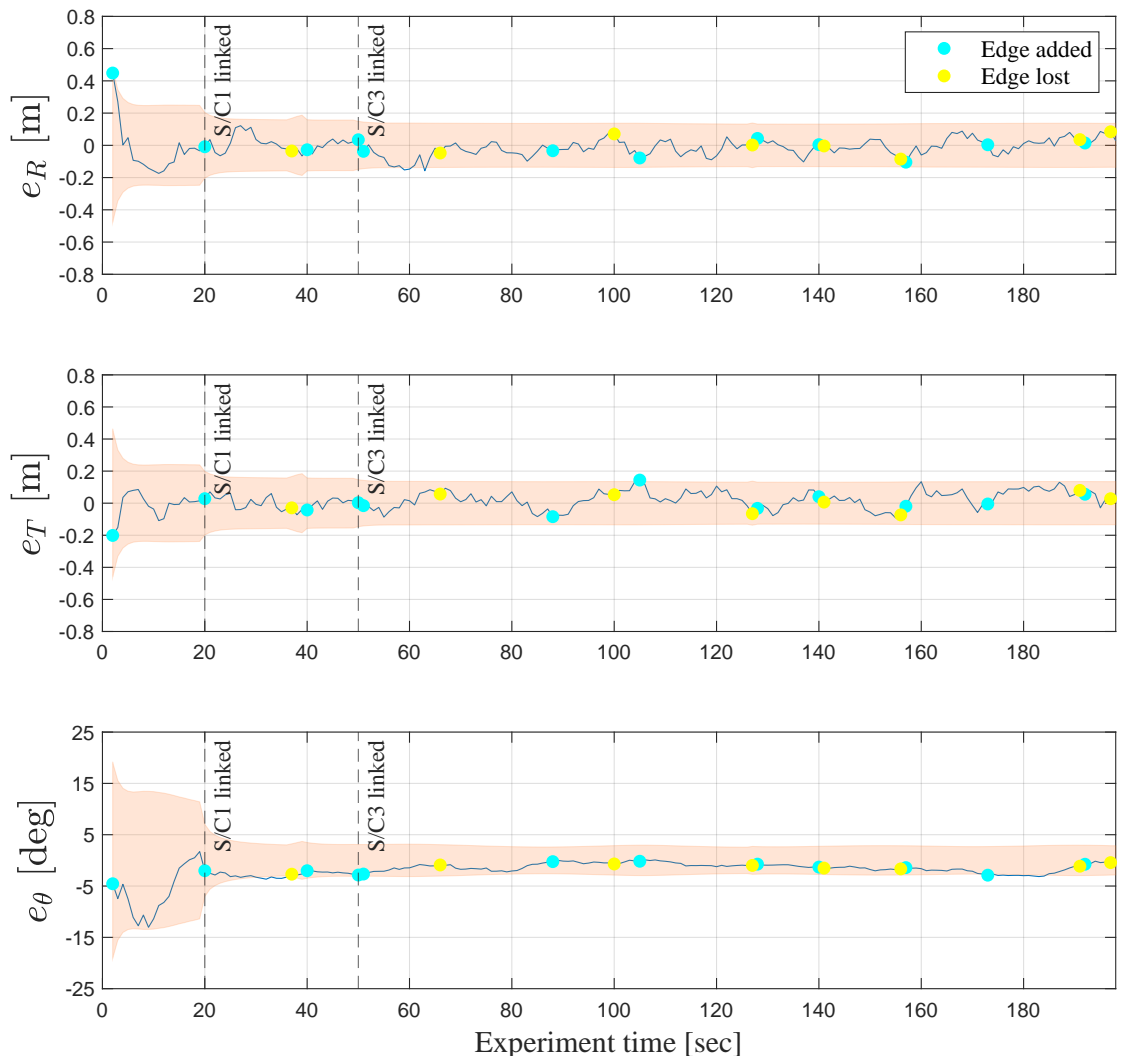

Figure 10: Estimation error of target (Spacecraft 4) by observer (Spacecraft 2)

\section{References}

Açıkmeşe, B., Mandić, M., Speyer, J.L., 2014. Decentralized observers with consensus filters for distributed discrete-time linear systems. Automatica 50, $1037-1052$.

Aragues, R., Carlone, L., Calafiore, G., Sagues, C., 2011. Multi-agent localization from noisy relative pose measurements, in: 2011 IEEE International Conference on Robotics and Automation, IEEE. pp. 364-369.

Bandyopadhyay, S., Chung, S.J., 2018. Distributed bayesian filtering using logarithmic opinion pool for dynamic sensor networks. Automatica 97, 7-17.

Bezouska, W., Barnhart, D., 2019. Decentralized cooperative localization with relative pose estimation for a spacecraft swarm, in: 2019 IEEE Aerospace Conference, IEEE. pp. 1-13. 
Blackmore, L., Hadaegh, F., 2009. Necessary and sufficient conditions for attitude estimation in fractionated spacecraft systems, in: AIAA Guidance, Navigation, and Control Conference, p. 6095.

Brown, O., Eremenko, P., Collopy, P., 2009. Value-centric design methodologies for fractionated spacecraft: Progress summary from phase i of the darpa system f6 program, in: AIAA Space 2009 Conference \& Exposition, p. 6540.

Capuano, V., Kim, K., Harvard, A., Chung, S.J., 2020. Monocular-based pose determination of uncooperative space objects. Acta Astronautica 166, 493506 .

Carlone, L., Ng, M.K., Du, J., Bona, B., Indri, M., 2010. Rao-blackwellized particle filters multi robot slam with unknown initial correspondences and limited communication, in: 2010 IEEE International Conference on Robotics and Automation, IEEE. pp. 243-249.

Cash, W., 2006. Detection of earth-like planets around nearby stars using a petal-shaped occulter. Nature 442, 51-53.

Cassinis, L.P., Fonod, R., Gill, E., 2019. Review of the robustness and applicability of monocular pose estimation systems for relative navigation with an uncooperative spacecraft. Progress in Aerospace Sciences 110.

Chung, S.J., Hadaegh, F.Y., 2011. Swarms of femtosats for synthetic aperture applications, in: Proceedings of the Fourth International Conference on Spacecraft Formation Flying Missions \& Technologies, St-Hubert, Quebec.

Chung, S.J., Paranjape, A.A., Dames, P., Shen, S., Kumar, V., 2018. A survey on aerial swarm robotics. IEEE Transactions on Robotics 34, 837-855.

De Jongh, W., Jordaan, H., Van Daalen, C., 2020. Experiment for pose estimation of uncooperative space debris using stereo vision. Acta Astronautica $168,164-173$.

Fourie, D., Tweddle, B.E., Ulrich, S., Saenz-Otero, A., 2014. Flight results of vision-based navigation for autonomous spacecraft inspection of unknown objects. Journal of Spacecraft and Rockets 51, 2016-2026.

Foust, R., Lupu, E.S., Nakka, Y.K., Chung, S.J., Hadaegh, F.Y., 2018. Ultrasoft electromagnetic docking with applications to in-orbit assembly, in: 69th International Astronautical Congress (IAC), Bremen, Germany.

Fox, D., Burgard, W., Kruppa, H., Thrun, S., 2000. A probabilistic approach to collaborative multi-robot localization. Autonomous Robots 8, 325-344.

Franceschelli, M., Gasparri, A., 2013. Gossip-based centroid and common reference frame estimation in multiagent systems. IEEE Transactions on Robotics $30,524-531$. 
Franchi, A., Oriolo, G., Stegagno, P., 2013. Mutual localization in multi-robot systems using anonymous relative measurements. The International Journal of Robotics Research 32, 1302-1322.

Garrido-Jurado, S., Muñoz-Salinas, R., Madrid-Cuevas, F.J., Marín-Jiménez, M.J., 2014. Automatic generation and detection of highly reliable fiducial markers under occlusion. Pattern Recognition 47, 2280-2292.

Gdoutos, E., Leclerc, C., Royer, F., Kelzenberg, M.D., Warmann, E.C., EspinetGonzalez, P., Vaidya, N., Bohn, F., Abiri, B., Hashemi, M.R., et al., 2018. A lightweight tile structure integrating photovoltaic conversion and rf power transfer for space solar power applications, in: 2018 AIAA Spacecraft Structures Conference, p. 2202.

Hadaegh, F.Y., Chung, S.J., Manohara, H.M., 2016. On development of 100gram-class spacecraft for swarm applications. IEEE Systems Journal 10, 673684 .

Hermann, R., Krener, A., 1977. Nonlinear controllability and observability. IEEE Transactions on Automatic Control 22, 728-740.

Kamal, A.T., Farrell, J.A., Roy-Chowdhury, A.K., 2012. Information weighted consensus, in: 2012 IEEE 51st IEEE Conference on Decision and Control (CDC), IEEE. pp. 2732-2737.

Kamal, A.T., Farrell, J.A., Roy-Chowdhury, A.K., 2013. Information weighted consensus filters and their application in distributed camera networks. IEEE Transactions on Automatic Control 58, 3112-3125.

Kekatos, V., Giannakis, G.B., 2012. Distributed robust power system state estimation. IEEE Transactions on Power Systems 28, 1617-1626.

Kelsey, J.M., Byrne, J., Cosgrove, M., Seereeram, S., Mehra, R.K., 2006. Visionbased relative pose estimation for autonomous rendezvous and docking, in: 2006 IEEE Aerospace Conference, IEEE. pp. 1-20.

Khan, U.A., Moura, J.M., 2008. Distributing the kalman filter for large-scale systems. IEEE Transactions on Signal Processing 56, 4919-4935.

López-Limón, C., Franceschelli, M., Seatzu, C., Ramírez-Treviño, A., 2014. A consensus algorithm for common reference frame estimation in networked multi-agent systems, in: 53rd IEEE Conference on Decision and Control, IEEE. pp. 6117-6122.

Marelli, D.E., Fu, M., 2015. Distributed weighted least-squares estimation with fast convergence for large-scale systems. Automatica 51, 27-39.

Markley, F.L., 2003. Attitude error representations for kalman filtering. Journal of Guidance, Control, and Dynamics 26, 311-317. 
Markley, F.L., Crassidis, J.L., 2014. Fundamentals of spacecraft attitude determination and control. volume 33. Springer, New York.

Martinelli, A., Pont, F., Siegwart, R., 2005. Multi-robot localization using relative observations, in: Proceedings of the 2005 IEEE International Conference on Robotics and Automation, IEEE. pp. 2797-2802.

Matsuka, K., Scharf, D., Filipe, N., Seubert, C., Bayard, D., 2019. Relative sensing, control precision, and mission delta-v trade-offs for precision formation flying in planetary orbit. Journal of Guidance, Control, and Dynamics , $1-15$.

Morgan, D., Chung, S.J., Blackmore, L., Acikmese, B., Bayard, D., Hadaegh, F.Y., 2012. Swarm-keeping strategies for spacecraft under j2 and atmospheric drag perturbations. Journal of Guidance, Control, and Dynamics 35, 14921506.

Morgan, D., Subramanian, G.P., Chung, S.J., Hadaegh, F.Y., 2016. Swarm assignment and trajectory optimization using variable-swarm, distributed auction assignment and sequential convex programming. The International Journal of Robotics Research 35, 1261-1285.

Nagavalli, S., Lybarger, A., Luo, L., Chakraborty, N., Sycara, K., 2014. Aligning coordinate frames in multi-robot systems with relative sensing information, in: 2014 IEEE/RSJ International Conference on Intelligent Robots and Systems, IEEE. pp. 388-395.

Nakka, Y.K., Foust, R.C., Lupu, E.S., Elliott, D.B., Crowell, I.S., Chung, S.J., Hadaegh, F.Y., 2018. Six degree-of-freedom spacecraft dynamics simulator for formation control research, in: 2018 AAS/AIAA Astrodynamics Specialist Conference, AIAA. pp. 1-20.

Olfati-Saber, R., Murray, R.M., 2004. Consensus problems in networks of agents with switching topology and time-delays. IEEE Transactions on Automatic Control 49, 1520-1533.

Opromolla, R., Fasano, G., Rufino, G., Grassi, M., 2017. A review of cooperative and uncooperative spacecraft pose determination techniques for closeproximity operations. Progress in Aerospace Sciences 93, 53-72.

Romano, M., Friedman, D.A., Shay, T.J., 2007. Laboratory experimentation of autonomous spacecraft approach and docking to a collaborative target. Journal of Spacecraft and Rockets 44, 164-173.

Roumeliotis, S.I., Bekey, G.A., 2002. Distributed multirobot localization. IEEE transactions on robotics and automation 18, 781-795.

Scharf, D.P., Hadaegh, F.Y., Ploen, S.R., 2003. A survey of spacecraft formation flying guidance and control (part i): Guidance, in: Proceedings of the American Controls Conference, Denver, Colorado. pp. 1733-1739. 
Schaub, H., Junkins, J.L., 2005. Analytical mechanics of space systems. American Institute of Aeronautics and Astronautics, Reston, Virginia. chapter Spacecraft Formation Flying.

Sullivan, J., Grimberg, S., DAmico, S., 2017. Comprehensive survey and assessment of spacecraft relative motion dynamics models. Journal of Guidance, Control, and Dynamics 40, 1837-1859.

Tweddle, B.E., Saenz-Otero, A., 2015. Relative computer vision-based navigation for small inspection spacecraft. Journal of Guidance, Control, and Dynamics 38, 969-978.

Yamanaka, K., Ankersen, F., 2002. New state transition matrix for relative motion on an arbitrary elliptical orbit. Journal of Guidance, Control, and Dynamics 25, 60-66.

Yan, Z., Jouandeau, N., Cherif, A.A., 2013. A survey and analysis of multirobot coordination. International Journal of Advanced Robotic Systems 10, 399 .

Zhang, G., Vela, P., Tsiotras, P., Cho, D.M., 2014. Efficient closed-loop detection and pose estimation for vision-only relative localization in space with a cooperative target, in: AIAA SPACE 2014 Conference and Exposition, p. 4262 .

Zhou, X.S., Roumeliotis, S.I., 2006. Multi-robot slam with unknown initial correspondence: The robot rendezvous case, in: 2006 IEEE/RSJ International Conference on Intelligent Robots and Systems, IEEE. pp. 1785-1792. 